\title{
Regulación interpersonal en el trabajo colaborativo: efectos en la comprensión lectora \\ y la autorregulación de estudiantes con diferentes estilos cognitivos
}

\author{
Interpersonal \\ Regulation in \\ Collaborative Learning: \\ Effects on Reading \\ Comprehension and \\ Self-regulation of \\ Students with Different \\ Cognitive Styles
}

\author{
Regulação interpessoal \\ na aprendizagem \\ colaborativa: efeitos na \\ compreensão da leitura \\ e na auto-regulação dos \\ alunos com diferentes \\ estilos cognitivos
}

Moreno-Caro, J., López-Vargas, 0. y SanabriaRodríguez, L. (2021). Regulación interpersonal en el trabajo colaborativo: efectos en la comprensión lectora y la autorregulación de estudiantes con diferentes estilos cognitivos. Folios, (54). https://doi.org/10.17227/folios.54-11403
Doctor en Educación. Profesor Universidad Pedagógica Nacional. Bogotá, Colombia. Correo: jomorenoc@pedagogica.edu.co

* Doctor en Educación. Profesor Universidad Pedagógica Nacional. Bogotá, Colombia. Correo: olopezv@pedagogica.edu.co

*** Doctor en Educación. Profesor Universidad Pedagógica Nacional. Bogotá, Colombia. Correo: lubsan@pedagogica.edu.co 


\title{
Resumen
}

En este artículo de investigación se reportan los resultados de un estudio cuasiexperimental que tuvo por objeto evaluar el impacto de un ambiente de aprendizaje computacional. Este proyecto apoyó la regulación social en el aprendizaje y la autorregulación de 166 estudiantes de Educación Media en una institución pública de la ciudad de Bogotá, Colombia. Mediante un diseño factorial 2×2, se exploraron las posibles diferencias entre dos modelos de regulación social y dos métodos de conformación grupal, teniendo en cuenta el estilo cognitivo de los sujetos en la dimensión dependencia-independencia de campo. Las pruebas no paramétricas implementadas, señalaron que el ambiente impactó significativamente la comprensión lectora, las creencias de control sobre el aprendizaje y la autorregulación metacognitiva de los participantes. Un Mancova no señaló diferencias significativas entre los grupos experimentales, luego de controlar el efecto de las covariables; no obstante, las tendencias en los datos indicaron que (a) regular la tarea benefició en mayor medida el logro y la autorregulación, en comparación con la regulación de la comunicación; (b) los grupos homogéneos alcanzaron mejores resultados que los grupos heterogéneos; $y$ (c) aunque los sujetos independientes alcanzaron mejores resultados que los sujetos dependientes de campo, el ambiente logró difuminar las diferencias significativas que existían entre ambos estilos cognitivos antes de la intervención, en términos del nivel inicial de comprensión lectora. Estos resultados sugieren que implementar soportes computacionales para apoyar la regulación de la tarea y la colaboración, puede beneficiar el logro y la autorregulación durante el trabajo colaborativo en la educación a distancia.

\section{Palabras clave}

aprendizaje colaborativo; instrucción por computador; regulación social; conformación grupal; comprensión lectora; estilo cognitivo

\begin{abstract}
This research article reports the results of a quasi experimental study that aimed to evaluate the impact of a computer-based learning environment that supported social regulation on learning achievement and self-regulation, implemented in 166 high school students of a public school in Bogotá. Using a 2x2 factorial design, possible differences between two models of social regulation and two methods of group formation were explored, considering the field dependence-independence cognitive styles. Non-parametric tests indicated that the computer support significantly impacted reading comprehension, control beliefs about learning, and metacognitive self-regulation in the students. A "MANCOVA" Reported no significant differences between the experimental groups, after controlling the effect of covariates; however, trends in data indicated that (a) task regulation benefited achievement and self-regulation more than communication regulation; (b) homogeneous groups achieved better results than heterogeneous groups; and (c) although independent subjects achieved better results than field-dependent subjects, the environment removed the significant differences that existed between both cognitive styles before the intervention, in terms of the initial level of reading comprehension. These results suggest that computer-based support for task and collaboration regulation can benefit achievement and self-regulation during collaborative learning in distance education.
\end{abstract}

\section{Keywords}

collaborative learning; computer instruction; social regulation; group formation; reading comprehension; cognitive style 


\section{Resumo}

Neste artigo apresentamos os resultados de um estudo quase experimental que visou avaliar o impacto de um ambiente de aprendizagem baseado em computador que apoiou a regulamentação social sobre o desempenho de aprendizagem e autoregulação de 166 alunos do Ensino Médio em uma instituição pública em Bogotá. Utilizando um desenho fatorial 2x2, foram exploradas possíveis diferenças entre dois modelos de regulação social e dois métodos de formação de grupos, levando em conta o estilo cognitivo dos sujeitos na dimensão da dependência de campo-independência. Testes não-paramétricos indicaram que o ambiente impactou significativamente a compreensão da leitura, crenças de controle sobre a aprendizagem e auto-regulação metacognitiva. Um “MANCovA" não relatou diferenças significativas entre os grupos experimentais, após 0 controle para o efeito de covariáveis; entretanto, tendências em dados indicou que 1) a regulação de tarefas beneficiou mais a realização e a auto-regulação do que a regulação da comunicação; 2) grupos homogêneos obtiveram melhores resultados que grupos heterogêneos; e 3) embora sujeitos independentes tenham obtido melhores resultados que sujeitos dependentes do campo, o ambiente conseguiu desfocar as diferenças significativas que existiam entre os dois estilos cognitivos antes da intervenção, em termos do nível inicial de compreensão de leitura. Estes resultados sugerem que os apoios informáticos à regulação das tarefas e da colaboração podem beneficiar a realização e a auto-regulação durante a aprendizagem em colaboração no ensino à distância.

\section{Palavras chave \\ aprendizagem colaborativa; ensino por computador; regulação social; construção de grupos; compreensão de leitura; estilo cognitivo}

\section{Introducción}

En el marco de las habilidades del siglo Xxi, la colaboración y la comunicación constituyen factores fundamentales que promueven la creación colectiva en diferentes esferas de la sociedad (Trilling y Fadel, 2009). No obstante, la colaboración en sí misma no garantiza el aprendizaje, menos aún al enfocarse en escenarios computacionales (Kirschner y Erkens, 2013). Para que la colaboración sea efectiva, los sujetos que trabajan desde este enfoque de aprendizaje requieren regular los comportamientos, las cogniciones, las emociones y las motivaciones propias, así como las de los compañeros (Rogat y Linnenbrink-Garcia, 2011). A la luz de esta necesidad, ha surgido el estudio de la regulación interpersonal del aprendizaje, paradigma que desde sus orígenes (McCaslin y Good, 1996) ha incrementado el volumen de investigaciones sobre el tema en los campos de la psicología y las tecnologías educativas (Hadwin et ál., 2017).

Pese a la continua indagación empírica sobre la regulación social en el trabajo colaborativo apoyado por computador, aún se desconoce el impacto diferencial que distintos modelos de regulación ( $\mathrm{p}$. ej., regulación de la tarea [Miller y Hadwin, 2015; Winne y Hadwin, 1998; 2008] o regulación de la colaboración y la comunicación [Saab et ál., 2007] tienen en el logro de aprendizaje y la propia autorregulación de los sujetos. En la misma línea se ubican los métodos de conformación grupal (Webb et ál., 1997) y el estilo cognitivo en la dimensión dependencia/independencia de campo (DIC) (Witkin y Goodenough, 1977), variables que, salvo contadas excepciones (López, 2010), han sido poco exploradas en el campo de la regulación social y el trabajo colaborativo apoyado por computador.

El logro de aprendizaje, por su parte, abarcó el nivel de comprensión lectora en estudiantes de educación media. El estudio propuesto se centró en este dominio específico de conocimiento, pues, pese a los esfuerzos gubernamentales por fortalecer las habilidades básicas de los estudiantes colombianos inscritos en los ciclos de educación básica y media (p. ej., el programa nacional Supérate y la estrategia distrital A-probar [MEN, 2019; SED, 2015]), los resultados obtenidos en pruebas internacionales señalan desempeños muy bajos, específicamente en lo que concierne al lenguaje y la comprensión lectora (OECD, 2015).

Los resultados arrojados por la prueba PISA 2015, ubicaron a Colombia en los últimos 14 lugares de los países evaluados. Según la Organización para la Cooperación y el Desarrollo Económico (OECD), más del $40 \%$ de los estudiantes colombianos se 
desempeñó por debajo del nivel 2 , en una escala de seis niveles de competencia lectora. En otras palabras, los estudiantes del país apenas logran hacer una conexión simple entre la información del texto y su conocimiento cotidiano, evidenciando dificultades para discernir la estructura y el propósito del texto, así como para localizar información específica en este (OECD, 2015). En tal sentido, es necesario generar alternativas instruccionales que fomenten el desarrollo de la comprensión lectora y la autorregulación en estudiantes de educación media, con base en la integración de las tecnologías educativas, el trabajo colaborativo y la regulación interpersonal del aprendizaje.

En este orden de ideas, hemos propuesto un estudio para evaluar el efecto que un ambiente computacional diseñado para apoyar la regulación social del aprendizaje tiene en la comprensión lectora y la capacidad autorreguladora de estudiantes de educación media que trabajan colaborativamente. El ambiente en cuestión permitió comparar dos modelos de regulación social (regulación de la tarea o regulación de la colaboración) dependiendo del método utilizado para la conformación de los grupos durante el trabajo colaborativo (grupos homogéneos o heterogéneos según el nivel inicial de comprensión lectora), así como su estilo cognitivo en la dimensión DIC.

\section{Referentes conceptuales}

\section{Aprendizaje colaborativo y conformación grupal}

El aprendizaje colaborativo ha sido definido como un esfuerzo coordinado para resolver conjuntamente una tarea, compartiendo un espacio de trabajo que integra metas, un estado inicial del problema, conocimientos sobre posibles acciones de resolución y asociaciones entre estos elementos (Roschelle y Teasley, 1995). El aprendizaje colaborativo tiene un impacto favorable en la cognición, la motivación y el comportamiento de los sujetos. Por ejemplo, promueve la construcción conjunta de conocimiento (Brindley et ál., 2009; Pea, 1994; Roschelle, 1992), favorece el logro académico y el pensamiento crítico (Chiong y Jovanovic, 2012; El-Deghaidy y Nouby, 2008; Garrison et ál., 2001; Gokhale, 1995), fortalece diferentes competencias de orden académico e incide positivamente en el aprendizaje autorregulado (Gilly, 1989; Kreijns et ál., 2003). Además, el aprendizaje colaborativo contribuye en la comunicación y el desarrollo de situaciones sociales que generan actitudes positivas hacia el aprendizaje (Gabbs, 2009; Lai, 2011).

Pese a las ventajas del trabajo colaborativo, algunos investigadores han señalado ciertos problemas que encara este enfoque de aprendizaje. Tal es el caso de las dificultades experimentadas por los sujetos cuando interactúan para solucionar un problema (Manlove et ál., 2006), situación que genera resistencia respecto a ser parte de un grupo (Brindley et ál., 2009). Asimismo, la participación en el trabajo colaborativo suele ser escasa y asimétrica (Jermann, 2004), convirtiéndose en un impedimento para alcanzar las metas propuestas (Chiong y Jovanovic, 2012). De otra parte, muchos sujetos desconocen cómo trabajar en equipo (The Metiri Group, 2009), haciendo que las interacciones tiendan a ser inusualmente complejas, reduciendo la efectividad del grupo colaborativo (Kreijns et ál., 2003).

De esta manera, los resultados de investigación en este campo tienden a ser contradictorios (Cohen, 1992; Dillenbourg et ál., 1995; Kreijns et ál., 2003). De hecho, el aprendizaje colaborativo depende de múltiples variables (p. ej., la composición de los grupos, la comunicación, las características de la tarea, etc.) y por consiguiente la colaboración en sí misma no garantiza resultados favorables (Dillenbourg, 1999). Para que esta sea efectiva se requiere de una interdependencia bien orquestada (Wasson, 1998), estructurando los procesos de aprendizaje o regulando las interacciones ocurridas (Dillenbourg, 2002). En otras palabras, el trabajo colaborativo debe apoyarse continuamente en la instrucción (Saab et ál., 2007), la retroalimentación (Lai, 2011) y la regulación de la colaboración (Jermann, 2004).

Ahora bien, la conformación de los grupos en el aprendizaje colaborativo (una de las variables ampliamente estudiada en el campo) puede darse 
en tres direcciones: a) los propios integrantes del grupo deciden su composición; b) el grupo se conforma a partir de una decisión ajena a sus integrantes, basada en criterios externos, a través de métodos de aleatorización simple o estratificada (p. ej., combinando aleatoriedad y homogeneidad) (Hilton y Phillips, 2010). Una tercera opción involucra la combinación de los anteriores métodos, una alternativa que favorece la asignación de los sujetos a condiciones experimentales, sin afectar el grado de empatía al interior del grupo: c) la conformación de grupos a cargo de los estudiantes, estableciendo unas condiciones preliminares que orienten la constitución grupal con base en el grado de homogeneidad requerido experimentalmente (p. ej., según la habilidad estudiada, el estatus social, el género, entre otros factores) (Pijls et ál., 2003). Al respecto cabe mencionar que el estudio propuesto abarcó este último método, involucrando el nivel inicial de comprensión lectora como criterio de homogenización para la conformación de los grupos durante el trabajo colaborativo apoyado por computador.

\section{Regulación interpersonal del aprendizaje}

La regulación desempeña un rol fundamental en el trabajo colaborativo, por cuanto este no implica necesariamente el aprendizaje, antes bien, requiere que los sujetos regulen tanto la solución de la tarea como la colaboración en sí misma (Hadwin et ál., 2011; Janssen et ál., 2012; Jermann, 2004; Miller y Hadwin, 2015; Volet et ál., 2009). En el primer caso, la regulación de actividades asociadas con la tarea abarca aspectos como la formulación de metas, la elección de estrategias, la planeación de acciones y la evaluación del progreso (Winne y Hadwin, 1998; 2008). En el segundo caso, la regulación de actividades relacionadas con la colaboración implica que los sujetos organicen sus interacciones en función de una comunicación efectiva (Saab et ál., 2007).

En la literatura sobre aprendizaje colaborativo apoyado por computador, la regulación generalmente se ha soportado en dos elementos: guiones y herramientas espejo (Miller y Hadwin, 2015). Los primeros consisten en instrucciones asociadas con la interacción, la colaboración y la solución conjunta del problema (O’Donnell y Dansereau, 1992). Por su parte, las segundas corresponden a mecanismos de concienciación sobre el conocimiento individual y colectivo que se tiene acerca de las situaciones y los hechos que emergen durante la colaboración. Tales procesos de concienciación pueden ser de tipo comportamental (acciones durante la colaboración), cognitivo (conocimientos y habilidades asociadas con la solución del problema) o social (grado de participación y empatía entre los participantes) (Bodemer y Dehler, 2011).

Los guiones ofrecen instrucciones prescriptivas sobre cómo regular el trabajo colaborativo, contemplando para ello los cuatro procesos incluidos por Winne y Hadwin $(1998,2008)$ en su modelo de autorregulación: definición de la tarea, formulación de metas y planes, ejecución de estrategias, y reflexión individual conducente a la adaptación metacognitiva. Las herramientas de concienciación grupal o herramientas de visualización, también llamadas herramientas espejo (Janssen y Bodemer, 2013), permiten a los sujetos monitorear su progreso y el de sus compañeros, en términos de la definición de la tarea, la formulación de metas y planes, y la ejecución de estrategias.

Ahora bien, en el marco de la regulación social, específicamente en ambientes colaborativos de aprendizaje, Hadwin et ál. (2017) precisan conceptual y teóricamente tres modos fundamentales de regulación, a saber: autorregulación, aprendizaje corregulado y regulación socialmente compartida. La autorregulación se refiere a la planeación, la representación de la tarea, la reflexión y la adaptación metacognitiva, estratégica y deliberada de un estudiante durante una actividad conjunta. Implica que los individuos asuman la responsabilidad sobre sus condiciones/estados cognitivos, comportamentales, motivacionales y emocionales en la medida que se necesite.

En palabras de Zimmerman (1989), la autorregulación corresponde al grado en que los estudiantes son participantes activos (en términos motivacionales, cognitivos y comportamentales) de sus propios procesos de aprendizaje. Siguiendo los planteamientos de Bandura (1986), Zimmerman 
sostiene que el aprendizaje autorregulado implica una causalidad recíproca entre tres factores: la persona, el comportamiento y el ambiente. Este enfoque da lugar a tres tipos de estrategias: regular procesos internos, cognitivos y metacognitivos, que varían según la especificidad disciplinar ( $p$. ej., las creencias individuales sobre su habilidad para resumir textos), regular el comportamiento (p. ej., planeando y eligiendo estrategias efectivas para solucionar una tarea específica), y regular el ambiente (p. ej., ajustando su entorno en función del ruido y la iluminación).

La regulación socialmente compartida implica las mismas demandas cognitivas, metacognitivas, emocionales y comportamentales de la autorregulación, pero enfocadas esta vez en el grupo, ya no solo en el individuo. Por consiguiente, este tipo de regulación implica que los grupos tomen control metacognitivo de la tarea a través de una negociación conjunta. Por su parte, el aprendizaje corregulado implica la estimulación transicional y flexible de la regulación, a través de las interacciones interpersonales. La corregulación, generada, guiada o dirigida por cualquier persona en el grupo, implica restricciones y oportunidades para que emerja el aprendizaje autorregulado o la regulación socialmente compartida (Hadwin et ál., 2017).

En este sentido, el estudio propuesto abarcó el diseño y la validación de un ambiente de aprendizaje para promover la regulación interpersonal durante la solución de problemas de lectura en lengua materna. Este ambiente involucró guiones y herramientas espejo para apoyar procesos de planeación conjunta al interior de cada grupo, así como procesos asociados con la ejecución de estrategias de comprensión lectora, el monitoreo y la adaptación metacognitiva a nivel individual y grupal. Ahora bien, entendiendo la regulación del aprendizaje como un sistema dinámico (Vauras y Volet, 2013); es decir, un escenario colaborativo en el que emergen los tres tipos de regulación descritos por Hadwin y sus colaboradoras (2017), se entiende que la interacción de los sujetos en el ambiente de aprendizaje propuesto involucre por igual la emergencia de dichos tipos de regulación.

\section{Comprensión lectora}

El estudio de la comprensión lectora se remonta a los orígenes de la cultura occidental, no obstante, es en pleno siglo xx cuando se le aborda como objeto de estudio desde disciplinas como la lingüística, la psicología cognitiva, la hermenéutica y los estudios del discurso. De acuerdo con la primera postura, la comprensión correspondía a un nivel de la lectura en el que se buscaba desentrañar el significado de un texto. En este sentido, la comprensión se organizaba jerárquicamente en habilidades lectoras relacionadas con la percepción de palabras, la descodificación o comprensión propiamente dicha, una respuesta emocional frente a lo leído y una evaluación del texto (Dubouis, 2000). Desde esta perspectiva se consideraba que el sentido de la lectura se encontraba únicamente en el texto, dejando a un lado los conocimientos y las experiencias del lector, cuyo papel consistía en extraer el significado unívoco del documento.

En oposición a este paradigma, disciplinas como la psicolingüística y la psicología cognitiva desplazaron el papel protagónico del texto hacia el lector. En este sentido, el énfasis fue puesto en los conocimientos y experiencias que le permiten a un lector comprender un texto (Grabe, 2003). Entendida de esta forma, la comprensión reposa en el procesamiento cognitivo de la información superficial y semántica del texto, al igual que en el almacenamiento de esta última, en razón del modelo mental que va construyendo el lector en su memoria (Kintsch y Van Dijk, 1983). Este enfoque, denominado interactivo, concibe la comprensión como un proceso en el que el lector construye en su mente un correlato del texto, a partir de la relación dinámica entre sus conocimientos y la información que el texto involucra. Este enfoque abarca procesos mentales que corresponden a los niveles lingüísticos del texto (Bower y Cirilo, 1985) y a los componentes para el almacenamiento y el procesamiento de la información (Rumelhart, 2003; Van Dijk, 1992). 
Desde este panorama, la lectura tiene como finalidad la comprensión y la producción de sentido, resultado de la interacción entre texto y lector en un contexto determinado. La lectura es entonces un proceso interactivo y dialéctico entre tres variables: a) el lector, sus conocimientos, objetivos y procesos cognitivos; b) el texto que incluye una serie de claves léxicas, formales, tipográficas y estructurales necesarias para su comprensión (Armbruster et ál., 1983); y c) el contexto expresado en tres dimensiones, una psicológica (intereses del lector), una textual (relación de enunciados) y una extratextual (marco espacio-temporal) (Tardif, 2003). Este proceso interactivo se estructura alrededor de dos fases, una física que corresponde a la descodificación (percepción física y reconocimiento de grafemas) y una fase intelectiva que abarca cuatro subprocesos: comprensión (identificación y reconstrucción del significado del texto), interpretación (juzgar, valorar y confrontar conocimientos y expectativas), retención (almacenar información y reestructurar esquemas cognitivos) y evocación (retomar la información para adelantar tareas) (Santiago et ál., 2010).

\section{Estilo cognitivo}

En el campo de la estilística educativa, el estilo cognitivo ha sido definido en relación con el término estilo en cuatro ámbitos, dos de los cuales son de especial interés para esta investigación: el de las ciencias de la conducta (expresión de la personalidad respecto a ciertas habilidades cognitivas) y el de la psicología (detalles característicos de la conducta que influyen en la consecución de una meta). Aquí, el estilo cognitivo se define a partir de cuatro rasgos fundamentales: a) un carácter diferenciador, pues, permite identificar una conducta individual; b) evidencia cierto grado de permanencia y por ende el estilo es estable; c) un atributo integrador pues incluye diferentes dimensiones del individuo; y c) un carácter neutral, es decir, no hay un estilo superior a otro (Hederich-Martínez, 2013).

La diversificación de estilos cognitivos en la literatura, producto de la indagación empírica y la revisión sistemática en torno al tema (Martens,
1975), abarcó inicialmente nueve dimensiones cognitivas asociadas con la forma en que una persona percibe, recuerda, piensa y resuelve problemas (Messick, 1969), a saber: dependencia/ independencia de campo; escaneo; categorización; conceptualización; complejidad/simplicidad cognitiva; reflexividad/impulsividad; nivelación/nitidez; control rígido/flexible; y tolerancia a experiencias irreales. Posteriormente, los investigadores propusieron modelos que agrupaban los estilos cognitivos según correspondieran a los procesos de percepción, memoria o pensamiento (Miller, 1987), pertenecieran al enfoque centrado en la cognición (Grigorenko y Sternberg, 1995) o abarcaran distintos niveles de complejidad y métodos de procesamiento (Nosal, 1990, citado por Kozhevnikov, 2007).

El estilo cognitivo en la dimensión dependencia/ independencia de campo (DIC) ha ocupado un lugar prominente en las anteriores clasificaciones. Este estilo cognitivo, entendido como la habilidad individual de desenmascarar información en tareas perceptuales vinculadas a la inteligencia espacial, abarca dos polaridades: una preferencia general por aprender de forma aislada (independencia de campo) en oposición a privilegiar la motivación extrínseca, una necesidad por seguir estructuras e indicaciones suministradas por el instructor (dependencia de campo) (Witkin y Asch, 1948).

Si bien la literatura sobre este estilo cognitivo generalmente ha clasificado los sujetos en alguna de estas dos polaridades, a partir de los resultados obtenidos en pruebas perceptuales como el embedded figure test (Volkmar, 2013), estudios recientes han optado por generar grupos intermedios (polaridad intermedia, si se quiere) a partir de la distribución de los datos en distintos percentiles (Hederich-Martínez y Camargo-Uribe, 2016; Hederich-Martínez et ál., 2016). En concordancia con estas investigaciones, el estudio aquí descrito involucró el análisis de los datos derivados del test de figuras enmascaradas (Hederich-Martínez y Camargo-Uribe, 1999), en función de distribuir la muestra en terciles: en el primer tercil (puntuaciones más bajas) se ubicaron los sujetos dependientes, en 
el segundo tercil se ubicaron los sujetos intermedios, mientras que en el tercero (puntuaciones más altas) se ubicaron los independientes de campo.

Ahora bien, varios estudios se han ocupado de explorar la relación entre el estilo cognitivo DIC y la lectura. Específicamente, la literatura al respecto ha señalado una fuerte asociación entre dicho estilo cognitivo y la comprensión lectora (Sabet y Mohammadi, 2013), teniendo en cuenta la velocidad y la ejecución de microprocesos lectores tales como la decodificación, el análisis sintáctico (Kirchner, 1987) y el análisis semántico (Camargo-Uribe y Hederich-Martínez, 2004). En esta dirección, las investigaciones han reportado que los sujetos independientes de campo obtienen mejores resultados en pruebas de lectura (Hederich-Martínez y Camargo-Uribe, 2016; Suh, 2009), especialmente cuando dichas pruebas demandan habilidades analíticas (Salmani-Nodousham, 2007), recuperación de información, uso de pistas contextuales y prueba de hipótesis (Sabet y Mohammadi, 2013).

\section{Método}

Esta investigación cuasiexperimental buscó determinar el impacto de un ambiente computacional diseñado para apoyar la regulación social, en el logro de aprendizaje y la autorregulación de estudiantes de educación media en una institución pública de Bogotá. Mediante un diseño factorial $2 \times 2$, se exploraron posibles diferencias entre dos modelos de regulación social (regular la tarea o regular la comunicación) y dos métodos de conformación grupal (grupos homogéneos o heterogéneos según el logro inicial), teniendo en cuenta el logro de aprendizaje (en términos de comprensión lectora individual) y el nivel de autorregulación (dado en los resultados que arrojó un cuestionario de autorreporte sobre el constructo en cuestión) que los estudiantes alcanzaron luego de la intervención. Además de las dos variables independientes arriba mencionadas, se incluyó como variable asociada al estilo cognitivo en la dimensión dependencia/ independencia de campo.

\section{Población y muestra}

En la investigación participaron todos los estudiantes matriculados en el ciclo de educación media de la institución educativa $(N=186)$. Tres criterios se tuvieron en cuenta para hacer más homogénea la muestra: primero, se excluyeron los estudiantes con discapacidad auditiva que formaban parte de la población $(n=11)$. Segundo, se excluyeron aquellos estudiantes que estaban cursando por segunda ocasión el mismo grado $(n=2)$, en la medida que ya habían participado en la prueba piloto del ambiente de aprendizaje, realizada el año anterior. Finalmente, se excluyeron los estudiantes que participaron en la primera etapa del estudio, pero que se retiraron de la institución antes de comenzar la implementación $(n=7)$. En suma, la muestra final correspondió a 166 sujetos, 73 hombres y 93 mujeres, con un rango de edad comprendido entre los 14 y 20 años $(M=16,35, S D=1,07)$.

\section{Materiales y mediciones}

\section{Ambiente de aprendizaje}

El ambiente de aprendizaje consistió en un curso de lectura colaborativa en modalidad b-learning, recurriendo al uso de tecnologías computacionales fuera y dentro del aula de clase (Graham, 2005). El curso se construyó en Moodle 3.2.1 y se alojó en el servidor de la Universidad Pedagógica Nacional, empleando principalmente cinco tipos de recursos: páginas, encuestas, chats, wikis y cuestionarios. El escenario computacional abarcó dos componentes, uno general para todos los estudiantes y otro con dos versiones distintas dependiendo de la condición experimental, esto es, los dos modelos de regulación. El componente general, módulo de dominio específico (apéndice A), se fundamentó en a) estrategias antes, durante y después de la lectura (Pressley, 2002); b) el enfoque de estrategias transaccionales para la lectura (Brown et ál., 1995); y c) el enfoque de codificación dual para representaciones mentales (Paivio, 1990).

El componente para apoyar la regulación social tuvo dos versiones, una para apoyar actividades rela- 
cionadas con la tarea y otra para apoyar actividades asociadas con la comunicación y la colaboración (apéndice B). En el primer caso, el apoyo se basó en los trabajos de Winne y Hadwin (1998; 2008) sobre autorregulación, así como en las propuestas teóricas y metodológicas de Miller y Hadwin (2015) para promover la regulación socialmente compartida (socially shared regulation -SSR-) en el aprendizaje colaborativo apoyado por computador. En el segundo caso, el soporte para apoyar la regulación de la comunicación y la colaboración, se fundamentó en el enfoque sociocultural, la cognición situada (Masterman y Sharples, 2002) y en algunos principios para favorecer los procesos comunicativos (respect, intelligent collaboration, deciding together y encouraging-RIDE-) durante el trabajo grupal (Saab et ál., 2005).

\section{Logro de aprendizaje}

El logro de aprendizaje se expresa en términos del nivel de comprensión lectora individual, teniendo en cuenta el componente de lectura en las pruebas PISA, componente que abarca cuatro dimensiones: recuperar e integrar información, reflexionar sobre el contenido de los textos, reflexionar sobre la forma de los textos, e interpretar los textos (OECD, 2010). Para determinar el nivel inicial de comprensión lectora en los sujetos, se empleó la prueba PISA 2006, versión reducida por Herrera y Pool (2007, citado por Cerchiaro et al., 2011). El logro de aprendizaje final se identificó a través de una prueba similar, compuesta por reactivos provenientes de la prueba PISA, versiones 2000, 2003, 2008 y 2009 (Cañón y Luna, 2011). La confiabilidad del instrumento se calculó teniendo en cuenta la fuerza de asociación entre los reactivos de ambas pruebas, pretest y postest $(r=0,96)$, además del alfa de Cronbach $(\alpha=0,82)$.

\section{Autorregulación}

El nivel de autorregulación en los sujetos se determinó con base en el cuestionario sobre estrategias motivadas para el aprendizaje (motivated strategies for learning questionnaire -MSLQ-, Pintrich et ál.,
1991), aplicado antes y después de la intervención pedagógica. Para los efectos particulares de este estudio se seleccionaron 42 reactivos distribuidos en las seis subescalas que abarcan exclusivamente la autorregulación: motivación intrínseca, valor de la tarea, creencias de control sobre el aprendizaje, autoeficacia, autorregulación metacognitiva y administración del tiempo y el ambiente de estudio (Hederich-Martínez et ál., 2016). La aplicación inicial del instrumento arrojó un alfa de Cronbach de 0,92 , en tanto la aplicación final dio lugar a un alfa de 0.90 .

\section{Estilo cognitivo en la dimensión dependencia/independencia de campo}

El estilo cognitivo de los sujetos se identificó mediante el test de figuras enmascaradas, instrumento desarrollado por Sawa (1966) a partir de los estudios de Witkin y Asch (1948) sobre orientación en el espacio y percepción en campos visuales. Esta prueba permite identificar el estilo cognitivo de los sujetos de acuerdo con su habilidad para encontrar una figura simple en otra más compleja (eficacia dada en el número de aciertos). En esta prueba, estandarizada por Hederich-Martínez y CamargoUribe (1999), un puntaje alto es un indicador de una tendencia hacia la independencia de campo, en tanto que un puntaje bajo señala una tendencia hacia la sensibilidad al medio. Según la literatura, esta prueba ha mostrado buenos niveles de confiabilidad en la población colombiana, dados en alfas de Cronbach que oscilan entre 0,91 y 0,97 (Hederich-Martínez, 2007).

\section{Procedimiento}

El estudio se desarrolló en tres etapas. En la primera se presentó la investigación a la comunidad educativa y se recogieron los consentimientos informados que registraron la participación voluntaria de los estudiantes (apéndice C). Se aplicaron las pruebas de entrada para determinar los niveles iniciales de comprensión lectora y autorregulación. A partir de los resultados de la prueba de lectura (logro inicial de aprendizaje), los estudiantes 
fueron clasificados en tres grupos: bajo nivel de comprensión lectora $(n=42)$, nivel intermedio $(n=82)$ y nivel alto $(n=42)$.

Posteriormente, los sujetos fueron asignados de forma aleatoria a una de las dos condiciones experimentales para apoyar la regulación social: regulación de actividades relacionadas con la tarea (ssr, $n=82$ ) y regulación de actividades asociadas con la comunicación y la colaboración (ride, $n=84$ ). Enseguida, los estudiantes fueron asignados aleatoriamente a una de las dos condiciones experimentales derivadas del método de conformación grupal: grupos homogéneos $(n=81)$ o heterogéneos $(n=85)$ de acuerdo con el nivel inicial de comprensión lectora.

La conformación de los grupos de trabajo colaborativo se basó en la estrategia de Pijls et ál. (2003), para evitar que la empatía entre los integrantes de cada grupo se convirtiera en una variable interviniente que afectara los resultados del estudio. En suma, se conformaron 50 grupos compuestos por 3 o 4 integrantes, grupos distribuidos equitativamente según el modelo de regulación social $(\mathrm{ssR}=25$, RIDE $=25)$ y el método de conformación grupal (24 grupos homogéneos y 26 heterogéneos).

La segunda etapa del estudio correspondió a la implementación del ambiente de aprendizaje. La interacción de los estudiantes con el escenario computacional se desarrolló durante nueve semanas académicas, dando lugar a un promedio de 30 sesiones de 55 minutos cada una. En modalidad presencial, los estudiantes utilizaron el ambiente en los laboratorios de idiomas, mientras que en modalidad no presencial, algunos grupos se conectaron en horarios extraescolares, adelantando por su cuenta algunas de las actividades del curso (apéndices A y B). En la tercera etapa se aplicaron las pruebas de salida respecto a las dos variables dependientes en el estudio (prueba PISA, componente de lectura, y cuestionario MSLQ), así como la prueba de figuras enmascaradas para determinar el estilo cognitivo de los sujetos en la dimensión dependencia-independencia de campo.

\section{Análisis de los datos}

Teniendo en cuenta el tipo de estudio y el diseño aquí propuestos, se eligió un análisis multivariado de covarianza (Mancova) como el método estadístico más adecuado para dar respuesta a los interrogantes de la investigación. Además del Mancova, se realizaron dos análisis complementarios: uno para determinar si existían diferencias significativas entre los grupos de cada variable independiente o asociada antes de la intervención; otro, emulando un diseño preexperimental pretest/postest sin grupo control (Campbell y Stanley, 2005), para identificar si existían diferencias entre los valores de las covariables (nivel inicial de comprensión lectora y autorregulación inicial) y los puntajes de las variables dependientes (logro final de aprendizaje y autorregulación final).

En relación con los análisis complementarios, se emplearon pruebas no paramétricas debido a que la cantidad de casos atípicos que arrojaron las pruebas de entrada excedía el $10 \%$ de la muestra y, por tanto, no era recomendable suprimir o transformar esa proporción de casos (Mertler y Vannatta, 2017). Por consiguiente, se utilizó el test U de Mann-Whitney para determinar si existían diferencias significativas entre los grupos de ambas variables independientes, en tanto el test Kruskall-Wallis permitió indagar acerca de posibles diferencias significativas entre los tres grupos de la variable asociada. Por su parte, la prueba de Wilcoxon Signed Rank se empleó para determinar si existían diferencias significativas en el logro de aprendizaje y la autorregulación, antes y después de la intervención. Cabe mencionar que todas las pruebas (paramétricas y no paramétricas) se realizaron en el programa IBM SPSS Statistics, versión 21.0.

\section{Resultados}

Mediante el estudio se buscó responder tres interrogantes: a) ¿existen diferencias entre los grupos experimentales antes de la intervención, en términos de comprensión lectora y autorregulación, teniendo en cuenta las tres variables independientes del estudio: 
modelo de regulación social, método de conformación grupal y estilo cognitivo DIC ${ }^{1}$ ?; b) ¿cuál es el efecto del ambiente de aprendizaje sobre las dos variables dependientes?; y c) ¿existen diferencias significativas en el logro de aprendizaje y el nivel final de autorregulación, entre los dos modelos de regulación social, los dos métodos de conformación grupal y las tres polaridades del estilo cognitivo, luego de controlar el efecto de las covariables logro y autorregulación iniciales?

Respecto al primer interrogante, se encontró que antes de la intervención existían diferencias significativas entre sujetos dependientes e independientes de campo, en términos de comprensión lectora inicial. Al explorar el segundo interrogante, se encontró un aumento significativo en la comprensión lectora, las creencias de control sobre el aprendizaje y la propia autorregulación de los sujetos. Por su parte, al abordar el tercer interrogante, los datos no señalaron diferencias significativas en términos del logro de aprendizaje y la autorregulación final, según el modelo de regulación social, el método de conformación grupal o el estilo cognitivo de los sujetos.

\section{Logro de aprendizaje y autorregulación iniciales}

Al explorar ambas covariables del estudio, únicamente se encontraron diferencias significativas entre los sujetos teniendo en cuenta su estilo cognitivo, a propósito del nivel inicial de comprensión lectora. En efecto, al considerar como hipótesis unilateral el hecho de que los sujetos independientes de campo suelen obtener mejores resultados en términos de logro de aprendizaje (HederichMartínez y Camargo-Uribe, 2000), la prueba $\mathrm{H}$ de Kruskal-Wallis señaló diferencias significativas al respecto (figura 1): (dependientes, $n=55$; intermedios, $n=59$; independientes, $n=52), \mathrm{X} 2(2$, $n=166)=5,59, p=0,034$ (unilateral). Al emplear el test U de Mann-Whitney para comparar parejas de estilos, se encontró que las diferencias significativas

1 Para los efectos del tratamiento estadístico de los datos, el diseño se transformó en $2 \times 2 \times 3$, involucrando al estilo cognitivo DIC como variable independiente, variable con tres valores: sujetos dependientes, intermedios e independientes de campo. emergían entre sujetos dependientes $(M d=44,23)$ e independientes de campo $(M d=50,0): U=1071,50$, $\mathrm{z}=-2,23, p=0,025$.

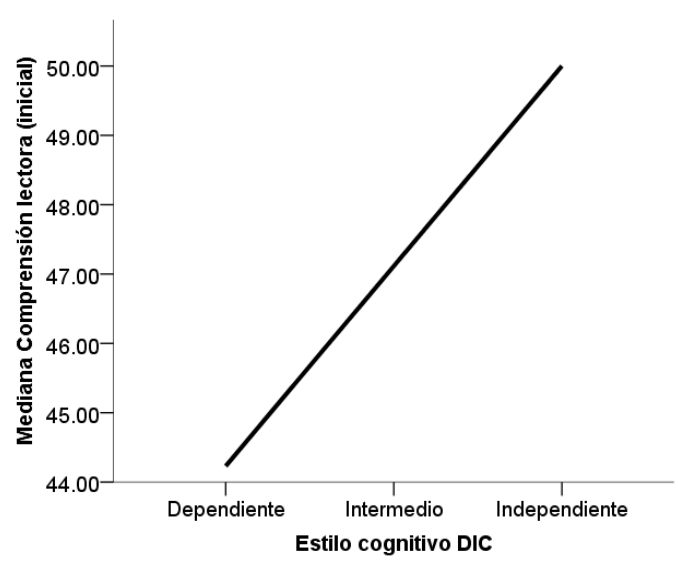

Figura 1. Comparación entre estilos cognitivos en la dimensión DIc: comprensión lectora inicial

Fuente: elaboración propia.

Estos resultados coinciden con los hallazgos reportados en estudios que han explorado el estilo cognitivo en ambientes naturales (Guisande et ál., 2007; Hederich-Martínez, 2007; Tinajero et ál., 2012; Witkin y Goodenough, 1977), así como en ambientes apoyados por computador (Angeli et ál., 2009; Chen y Macredie, 2002; Handal y Herrington, 2004; Weller et ál., 1994). En efecto, los resultados de estas investigaciones señalan reiteradamente que los sujetos independientes de campo suelen desempeñarse mucho mejor en la mayoría de ámbitos disciplinares, en comparación con sujetos intermedios $\mathrm{y}$, fundamentalmente, dependientes de campo.

\section{Efecto del ambiente sobre el logro de aprendizaje y la autorregulación final}

La prueba de Wilcoxon reveló diferencias significativas entre el nivel de comprensión lectora medido antes de la intervención y al finalizar la misma (figura 2): $z=-8,393, p<0,001$, con un tamaño del efecto medio $(r=0,46)$. Estos resultados indican que el logro de aprendizaje luego de la intervención $(M d=61,90)$ fue significativamente 
superior al alcanzado por los sujetos antes de la $\operatorname{misma}(M d=47,12)$.

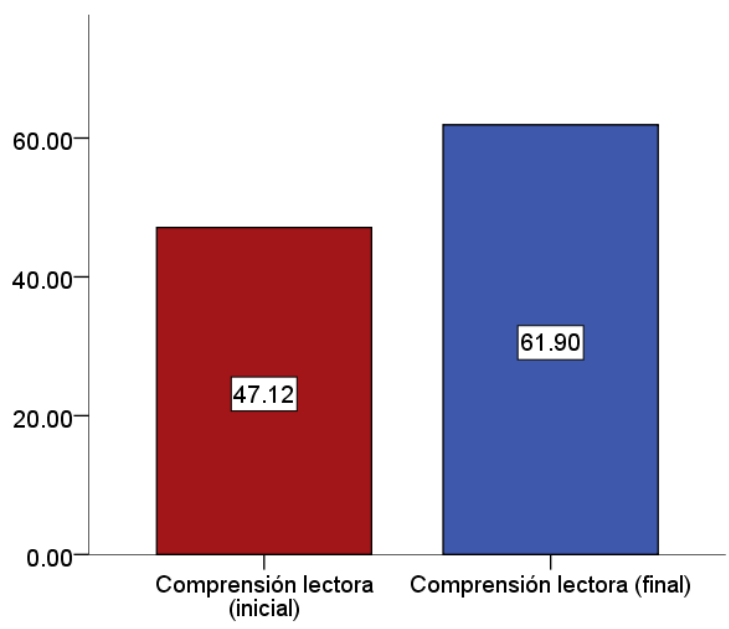

Figura 2. Mediana de las puntuaciones obtenidas en las pruebas de lectura aplicadas antes y después de la intervención pedagógica

Fuente: elaboración propia.

Estos resultados respaldan las investigaciones que han reportado correlaciones significativas entre la regulación social y el desempeño alcanzado por grupos que trabajan colaborativamente (Janssen et ál., 2012; Järevelä et ál., 2013; Järvelä et ál., 2016; Volet et ál., 2009). En este sentido, los efectos de la intervención sugieren que combinar el desarrollo de estrategias en un dominio específico de conocimiento con soportes computacionales para apoyar la regulación social (bien sea de la tarea o de la colaboración), favorece el logro de aprendizaje. En la misma línea, los resultados corresponden con las investigaciones que han empleado soportes computacionales para apoyar la regulación social, principalmente cuando se enfocan en la regulación de hábitos de estudio y actividades asociadas con la tarea (Hwang et ál., 2015; Lin, 2018; Saab et ál., 2007; Zheng y Huang, 2016).

En relación con el efecto de la intervención sobre la autorregulación de los sujetos, la prueba de Wilcoxon reveló diferencias significativas entre las creencias de control sobre el aprendizaje, medidas antes de la intervención $(M d=5,25)$ y después de finalizada $(M d=5,50): z=-2,950, p<0,01$ (figura
3). Los mismos resultados emergieron al comparar la autorregulación metacognitiva antes $(M d=4,50)$ y después de la intervención $(M d=4,67): z=-2,928$, $p<0,01$ (figura 4).

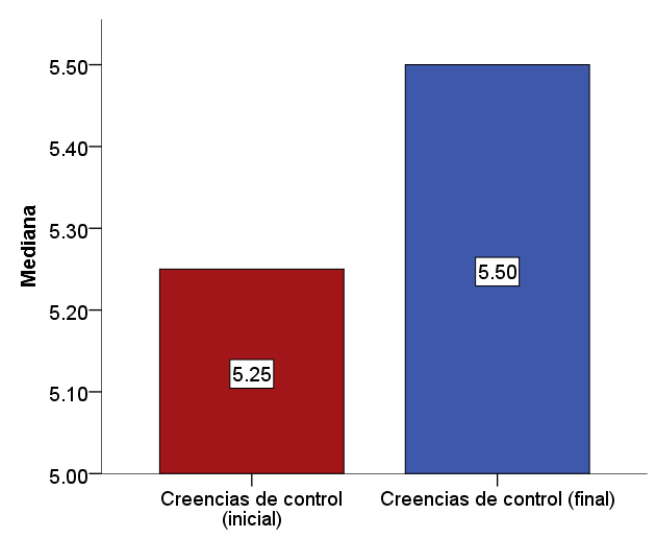

Figura 3. Mediana de las puntuaciones obtenidas en creencias de control sobre el aprendizaje, antes y después de la intervención pedagógica

Fuente: elaboración propia.

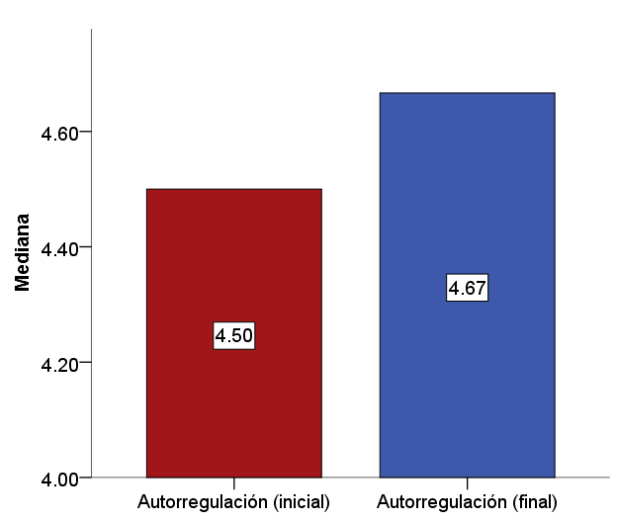

Figura 4. Mediana de las puntuaciones obtenidas en autorregulación, antes y después de la intervención pedagógica

Fuente: elaboración propia.

A propósito del efecto que el ambiente tuvo en las creencias de control sobre el aprendizaje, los resultados sugieren que los estudiantes comprendieron que los resultados de su aprendizaje se deben a sus propios esfuerzos antes que a factores externos; por ende, al asumir que tienen control sobre su desempeño, los estudiantes comprendieron la efectividad 
de estudiar estratégicamente. Este valor agregado se reflejó sobre la otra escala metacognitiva en la que el ambiente impactó de manera significativa, esto es, la propia regulación metacognitiva. En esta dirección, los estudiantes progresaron significativamente respecto a tres procesos clave en el marco de la autorregulación: la planeación, el monitoreo y la propia regulación, es decir, la capacidad de calibrar comportamientos, cogniciones y emociones, en función de alcanzar las metas de aprendizaje y mejorar el desempeño (Pintrich et ál., 1991).

\section{Resultados del análisis} multivariado de covarianza

Antes de verificar los supuestos para correr el Mancova, se identificaron datos atípicos (outliers) en el interior de los grupos experimentales y los grupos derivados del estilo cognitivo DIC como variable asociada. Para identificar datos atípicos univariados se emplearon estadísticos descriptivos, diagramas de caja y diagramas de tallo y hojas. Por su parte, la identificación de datos atípicos multivariados se basó en la distancia de Mahalanobis (Tabanachi y Fidell, 2014).

Después de identificar valores atípicos univariados, se procedió a reducir su impacto en los resultados del análisis, eliminando los casos que pre- sentaban el mayor número de outliers en la muestra $(n=2)$ y transformando los valores atípicos restantes $(n=8)$ mediante la técnica winzorinzing (Shete et ál., 2004). En cuanto los outliers multivariados, no se encontraron distancias de Mahalanobis superiores a las puntuaciones límite, según los grados de libertad asociados con la cantidad de variables dependientes y covariables.

En suma, luego de remover los dos casos que presentaban el mayor número de valores atípicos, así como otro caso que involucró datos perdidos en términos del nivel inicial de autorregulación, la muestra resultante para efectuar el análisis multivariado de covarianza comprendió un total de 163 casos.

\section{Efecto de las variables independientes y las covariables sobre la variable dependiente combinada}

La prueba de contrastes multivariados (tabla 1) señaló que la única covariable que incidió significativamente en la variable dependiente combinada fue la comprensión lectora inicial (lecPreTr) $[\lambda$ de Wilks $=0,795, F(7,138)=5,093, p<0,001, \eta^{2}$ multivariado $=0,205]$. Por el contrario, las covariables relacionadas con la autorregulación inicial, las variables independientes y la variable asociada, no incidieron significativamente en la variable dependiente combinada.

Tabla 1. Contrastes multivariados ${ }^{a}$

\begin{tabular}{|c|c|c|c|c|c|c|}
\hline Efecto & Lambda de Wilks & $\mathbf{F}$ & $\begin{array}{l}\text { GI de la } \\
\text { hipótesis }\end{array}$ & $\begin{array}{l}\text { Gl del } \\
\text { error }\end{array}$ & Sig. & Eta al cuadrado parcial \\
\hline Intersección & 0,394 & $30,305^{b}$ & 7 & 138 & 0,000 & 0,606 \\
\hline lecPreTr & 0,795 & $5,093^{b}$ & 7 & 138 & 0,000 & 0,205 \\
\hline motintPre & 0,942 & $1,207^{\mathrm{b}}$ & 7 & 138 & 0,303 & 0,058 \\
\hline valTaPre & 0,955 & $0,923^{b}$ & 7 & 138 & 0,491 & 0,045 \\
\hline creConPre & 0,941 & $1,225^{b}$ & 7 & 138 & 0,293 & 0,059 \\
\hline autoEfiPre & 0,938 & $1,305^{b}$ & 7 & 138 & 0,252 & 0,062 \\
\hline autoRegPre & 0,971 & $0,597^{b}$ & 7 & 138 & 0,758 & 0,029 \\
\hline adTiEsPre & 0,918 & $1,755^{b}$ & 7 & 138 & 0,101 & 0,082 \\
\hline Apoyo & 0,915 & $1,831^{\mathrm{b}}$ & 7 & 138 & 0,086 & 0,085 \\
\hline formGrup & 0,944 & $1,180^{\mathrm{b}}$ & 7 & 138 & 0,318 & 0,056 \\
\hline
\end{tabular}


Universidad Pedagógica Nacional

Facultad de Humanidades

\begin{tabular}{|c|c|c|c|c|c|c|}
\hline Ecdic & 0,846 & $1,722^{\mathrm{b}}$ & 14 & 276 & 0,051 & 0,08 \\
\hline $\begin{array}{l}\text { apoyo * form- } \\
\text { Grup }\end{array}$ & 0,96 & $0,817^{b}$ & 7 & 138 & 0,574 & 0,04 \\
\hline apoyo * ecdic & 0,958 & $0,430^{\mathrm{b}}$ & 14 & 276 & 0,964 & 0,021 \\
\hline $\begin{array}{l}\text { formGrup * ec- } \\
\text { dic }\end{array}$ & 0,902 & $1,049^{b}$ & 14 & 276 & 0,405 & 0,051 \\
\hline $\begin{array}{l}\text { apoyo * form- } \\
\text { Grup * ecdic }\end{array}$ & 0,925 & $0,788^{b}$ & 14 & 276 & 0,682 & 0,038 \\
\hline
\end{tabular}

a Diseño: Intersección + lecPreTr + motlntPre + valTaPre + creConPre + autoEfiPre + autoRegPre + adTiEsPre + apoyo

+ formGrup + ecdic + apoyo * formGrup + apoyo * ecdic + formGrup * ecdic + apoyo * formGrup * ecdic

${ }^{\mathrm{b}}$ Estadístico exacto

Fuente: elaboración propia.

\section{Efecto del soporte computacional para apoyar la regulación social en el logro de aprendizaje y la autorregulación de los sujetos}

La prueba de los efectos intersujetos (tabla 2) correspondiente al soporte computacional para apoyar la regulación social (apoyo), indicó que esta variable independiente solo afectó significativamente la autorregulación final de los sujetos $[F(1,144)=3,946$, $p=0,046, \eta^{2}$ parcial $\left.=0,027\right]$. No obstante, al modifi- car el nivel de alfa con base en el ajuste de Bonferroni (alfa de 0,05 a 0,007), se encontró que esta variable independiente no incidió significativamente en el nivel de autorregulación final, hecho que respalda el bajo tamaño del efecto reportado, pues el soporte computacional (regulación de la tarea vs. regulación de la colaboración) solo habría explicado el $2 \%$ de la varianza en la variable dependiente.

Tabla 2. Prueba de los efectos intersujetos para la variable independiente soporte computacional para apoyar la regulación social

\begin{tabular}{|c|c|c|c|c|c|c|c|}
\hline Origen & Variable dependiente & $\begin{array}{c}\text { Suma de } \\
\text { cuadrados } \\
\text { tipo III }\end{array}$ & gl & $\begin{array}{c}\text { Media } \\
\text { cuadrática }\end{array}$ & $F$ & Sig. & $\begin{array}{c}\text { Eta al } \\
\text { cuadrado } \\
\text { parcial }\end{array}$ \\
\hline \multirow{7}{*}{ 条 } & Comprensión lectora (final) & 372,478 & 1 & 372,478 & 1,896 & 0,171 & 0,013 \\
\hline & Motivación (final) & 1,868 & 1 & 1,868 & 2,991 & 0,086 & 0,02 \\
\hline & Valor de la tarea (final) & 0,754 & 1 & 0,754 & 1,816 & 0,18 & 0,012 \\
\hline & Creencias de control (final) & 0,429 & 1 & 0,429 & 0,765 & 0,383 & 0,005 \\
\hline & Autoeficacia (final) & 0,018 & 1 & 0,018 & 0,046 & 0,831 & 0,000 \\
\hline & Autorregulación (final) & 1,722 & 1 & 1,722 & 3,946 & 0,049 & 0,027 \\
\hline & Administración de T y A (final) & 0,000 & 1 & 0,000 & 0,000 & 0,983 & 0,000 \\
\hline
\end{tabular}

Fuente: elaboración propia. 
En función de estos resultados, algunos estudios han encontrado que la regulación social no necesariamente beneficia el logro o incluso la propia autorregulación. Por ejemplo, Schoor y Bannert (2012) no encontraron diferencias significativas entre díadas con altos y bajos desempeños, en términos de las frecuencias de las actividades reguladoras acaecidas durante la colaboración. Una situación análoga fue reportada por Perera et ál. (2009), quienes tampoco encontraron relaciones significativas entre el desempeño y las frecuencias y secuencias de las actividades realizadas por grupos fuertes o débiles durante el trabajo colaborativo en línea.

En la misma dirección se sitúan, a propósito de los soportes computacionales, algunos estudios que han recurrido a los guiones y las herramientas de concienciación para apoyar la regulación social. En el primer caso, los guiones no necesariamente favorecen el logro, por cuanto suelen dificultar la colaboración al forzar a los sujetos a interactuar de forma poco natural, al secuenciar linealmente las tareas, afectando negativamente aquellos estudiantes que prefieren abordar los problemas de forma holística, e incluso al incrementar la carga cognitiva, demandando esfuerzos adicionales para comprender y utilizar los propios guiones (Dillenbourg, 2002; Panadero et ál., 2015). En el caso de las herramientas de concienciación, algunos estudios han reportado que este tipo de apoyo computacional tampoco ha impactado significativamente el logro de aprendizaje, argumentando que dichas herramientas se centran en reflexiones retrospectivas, antes que en la formulación de planes, esto es, reflexiones prospectivas conducentes a posibles adaptaciones metacognitivas (Kirschner et ál., 2014).

Aunque el escenario computacional impactó positivamente en el logro de aprendizaje y la autorregulación de los sujetos (ver sección "Efecto del ambiente sobre el logro de aprendizaje y la autorregulación final"), no hubo diferencias significativas entre los participantes dependiendo del tipo de soporte utilizado para apoyar la regulación (tabla 2). Tal como se ha mencionado, estos resultados pueden explicarse a la luz de los estudios previos sobre el tema, sin embargo, ¿cómo precisar dichas explicaciones en esta investigación en particular? Por una parte, el hecho de que en algunos estudios no se haya encontrado correlación entre la regulación y el logro (Perera et ál., 2009; Schoor y Bannert, 2012) puede deberse a la complejidad de la tarea (Kirschner, Paas y Kirschner, 2011) y al dominio específico de conocimiento (Zimmerman, 1989).

En el primer caso, las tareas propuestas en el ambiente de aprendizaje correspondieron a problemas altamente estructurados (cuestionarios con preguntas de selección múltiple), actividades que probablemente no demandaron elevados niveles de regulación por parte de los participantes. En el segundo caso, el nivel de autorregulación depende de la experticia de los sujetos en determinado dominio específico de conocimiento, por ende, el hecho de que ambas condiciones experimentales tuvieran en común el módulo para apoyar la comprensión lectora (apéndice A) podría haber incidido en que no emergieran diferencias significativas entre los dos soportes para apoyar la regulación, en suma, habría tenido más peso el componente disciplinar que el componente regulador.

De otra parte, las investigaciones en el campo han señalado efectos heterogéneos de los guiones y las herramientas de concienciación, sobre el logro y la regulación de los sujetos. Mientras que algunos estudios apelan por las bondades de dichas herramientas (Lin, 2018; Su et ál., 2018; Zheng y Huang, 2016), otras investigaciones han reportado resultados menos favorables (Kirschner et ál., 2014; Panadero et ál., 2015). Por consiguiente, el que los soportes computacionales en este estudio emplearan ambos recursos, pudo incidir en el hecho que no se presentaran diferencias significativas entre ambas condiciones experimentales. En efecto, tanto la versión del ambiente para apoyar la regulación de la tarea (SSR), como la versión para apoyar la comunicación y la colaboración (RIDE), recurrieron a guiones para orientar procesos de planeación y reflexión, así como a herramientas de concienciación para apoyar el monitoreo, la definición de la tarea y la propia solución de los problemas de lectura (apéndice B). 
Universidad Pedagógica Nacional

Facultad de Humanidades

\section{Efecto del método de conformación grupal en el logro de aprendizaje y el nivel de autorregulación de los sujetos}

La prueba de los efectos intersujetos (tabla 3) ejecutada sobre el método de conformación grupal (formGrup), indicó que esta variable independiente no incidió de forma significativa en ninguna de las variables dependientes contempladas en el estudio. Estos resultados contradicen gran parte de la literatura sobre métodos de conformación grupal, investigaciones que abogan por la superioridad de los grupos heterogéneos sobre los homogéneos (Johnson y Johnson, 1989; Kagan y Kagan, 2009), principalmente en lo que respecta al logro de aprendizaje (Webb, Nemer y Chizhik, 1997). En esta dirección, suele afirmarse que los grupos heterogéneos favorecen mucho más a los sujetos con bajos niveles de conocimientos previos (Zhang, Kalyuga, Lee y Lei, 2016), en tanto la homogeneidad grupal no beneficia el logro de aprendizaje cuando los colectivos están compuestos exclusivamente por sujetos expertos (Kirschner, Sweller, Kirschner y Zambrano, 2018).

Tabla 3. Prueba de los efectos intersujetos para la variable independiente método de conformación grupal

\begin{tabular}{|c|c|c|c|c|c|c|c|}
\hline Origen & Variable dependiente & $\begin{array}{l}\text { Suma de } \\
\text { cuadrados } \\
\text { tipo III }\end{array}$ & GI & $\begin{array}{l}\text { Media cua- } \\
\text { drática }\end{array}$ & $\mathbf{F}$ & Sig. & $\begin{array}{c}\text { Eta al } \\
\text { cuadrado } \\
\text { parcial }\end{array}$ \\
\hline \multirow{7}{*}{ 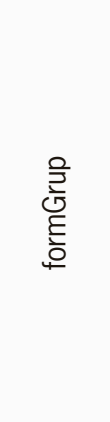 } & Comprensión lectora (final) & 104,824 & 1 & 104,824 & 0,534 & 0,466 & 0,004 \\
\hline & Motivación (final) & 0,364 & 1 & 0,364 & 0,584 & 0,446 & 0,004 \\
\hline & Valor de la tarea (final) & 0,101 & 1 & 0,101 & 0,244 & 0,622 & 0,002 \\
\hline & Creencias de control (final) & 0,667 & 1 & 0,667 & 1,19 & 0,277 & 0,008 \\
\hline & Autoeficacia (final) & 0,635 & 1 & 0,635 & 1,588 & 0,21 & 0,011 \\
\hline & Autorregulación (final) & 0,004 & 1 & 0,004 & 0,01 & 0,922 & 0,000 \\
\hline & Admin. de T y A (final) & 0,029 & 1 & 0,029 & 0,064 & 0,801 & 0,000 \\
\hline
\end{tabular}

Fuente: elaboración propia.

Por el contrario, los resultados de nuestro estudio señalaron que el ambiente de aprendizaje afectó por igual a ambos tipos de grupos, llegando incluso a beneficiar en mayor medida (aunque no de forma significativa) a los grupos homogéneos, grupos que obtuvieron mejores resultados en el logro de aprendizaje y en la mayoría de escalas de autorregulación (ver sección "Estadísticos descriptivos respecto a las variables independientes"). Estos resultados señalan el potencial del ambiente propuesto para compensar las desventajas de los grupos homogéneos en términos de restructuración y afinación del aprendizaje, en comparación con los grupos heterogéneos (Rumelhart y Norman, 1978). Por supuesto, estos resultados no son tan extraños en la literatura sobre el tema, pues, coinciden con los hallazgos reportados en otras investigaciones (Falk y Johnson, 1977; Fiedler, Meuwese y Oonk, 1961).
Al respecto emergen varias explicaciones, en primer lugar, los estudios previos han demostrado que los grupos heterogéneos son más exitosos entre hombres, pero no entre mujeres (Ziller, 1955); teniendo en cuenta que el criterio para conformar los grupos en nuestro estudio correspondió al nivel inicial de comprensión lectora y no al sexo de los sujetos, sería comprensible entonces que los grupos heterogéneos no hubiesen superado a los grupos homogéneos. En segundo lugar, el hecho de que los propios estudiantes conformaran sus grupos pudo incidir positivamente en su desempeño y autorregulación (Brindley et ál., 2009; Juwah, 2006). En tercer lugar, los sujetos que integraron los grupos homogéneos coincidían en términos de habilidades y estándares asociados con su nivel de comprensión lectora, condición que pudo favorecer la metacognición compartida en dichos grupos (Malmberg et ál., 2017; Winne, 2015). 


\section{Incidencia del estilo cognitivo en el logro de aprendizaje y el nivel de autorregulación}

La prueba de los efectos intersujetos (tabla 4) para el estilo cognitivo dic, señaló que esta variable asociada no incidió significativamente en ninguna de las variables dependientes del estudio. De hecho, pese a que el efecto del estilo cognitivo sobre las creencias de control parecía estar al límite del nivel de significación $[F(2,144)=2,898, p=0,058$, $\eta^{2}$ parcial $\left.=0,039\right]$, luego de aplicar el ajuste de Bonferroni, el posible efecto de esta variable asociada desaparece por completo.

Tabla 4. Prueba de los efectos intersujetos para la variable asociada estilo cognitivo en la dimensión dependencia/independencia de campo

\begin{tabular}{|c|c|c|c|c|c|c|c|}
\hline Origen & Variable dependiente & $\begin{array}{c}\text { Suma de } \\
\text { cuadrados } \\
\text { tipo III }\end{array}$ & gl & $\begin{array}{l}\text { Media cua- } \\
\text { drática }\end{array}$ & $\mathbf{F}$ & Sig. & $\begin{array}{c}\text { Eta al } \\
\text { cuadrado } \\
\text { parcial }\end{array}$ \\
\hline \multirow{7}{*}{$\stackrel{\cup}{\circ}$} & Comprensión lectora (final) & 940,243 & 2 & 470,121 & 2,393 & 0,095 & 0,032 \\
\hline & Motivación (final) & 1,731 & 2 & 0,866 & 1,386 & 0,253 & 0,019 \\
\hline & Valor de la tarea (final) & 0,648 & 2 & 0,324 & 0,779 & 0,461 & 0,011 \\
\hline & Creencias de control (final) & 3,249 & 2 & 1,624 & 2,898 & 0,058 & 0,039 \\
\hline & Autoeficacia (final) & 0,312 & 2 & 0,156 & 0,391 & 0,677 & 0,005 \\
\hline & Autorregulación (final) & 0,086 & 2 & 0,043 & 0,098 & 0,907 & 0,001 \\
\hline & Admin. de T y A (final) & 0,558 & 2 & 0,279 & 0,611 & 0,544 & 0,008 \\
\hline
\end{tabular}

Fuente: elaboración propia.

Estos resultados indican que el ambiente de aprendizaje difuminó la distancia que existía inicialmente entre los sujetos independientes y los dependientes de campo respecto al logro de aprendizaje. Los hallazgos coinciden con otras investigaciones que han conseguido reducir la distancia entre estilos cognitivos mediante apoyos computacionales (López et ál., 2012; Zehavi, 1995). Una posible explicación de estos resultados deriva de las herramientas empleadas en nuestro ambiente de aprendizaje, guiones y herramientas de concienciación. En efecto, otros estudios han demostrado que utilizar preguntas (guiones) y mecanismos de retroalimentación favorecen distintos estilos cognitivos (Myint, 1996; Pi-Sui-Hsu y Dwyer, 2004). Más aún, investigaciones en el área han demostrado que la implementación de estrategias afectivas, sociales (p. ej., las incluidas en nuestra versión RIDE) y cognitivas (p. ej., las incluidas en nuestra versión SSR) favorecen especialmente a los sujetos dependientes de campo (p. ej., Altun y Cakan, 2006).

\section{Estadísticos descriptivos respecto a las variables independientes}

Aunque el Mancova no arrojó diferencias significativas entre los modelos de regulación social ni entre los métodos de conformación grupal involucrados en el estudio, se examinaron los estadísticos descriptivos $y$ las medias marginales estimadas para identificar posibles tendencias en la distribución de los datos. Al explorar el soporte computacional para apoyar la regulación social, en relación con las tres variables que mejoraron significativamente luego de la intervención (figura 5), se encontró que la condición con apoyo para regular actividades asociadas con la tarea (ssr) generó mejores resultados en términos del logro de aprendizaje $(M=63,17, E T=1,60)$, las creencias de control sobre el aprendizaje $(M=5,54, E T=0,08)$ y la autorregulación metacognitiva $(M=4,79, E T=0,07)$. 
Universidad Pedagógica Nacional

Facultad de Humanidades

Comprensión lectora
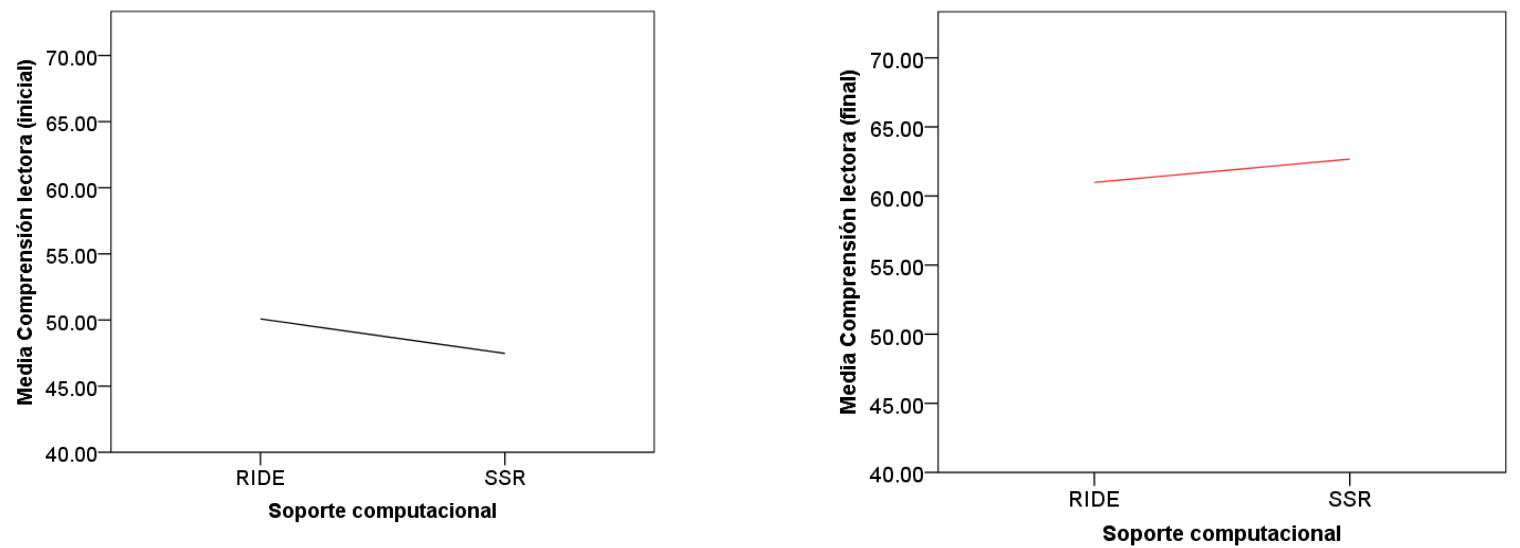

Creencias de control sobre el aprendizaje
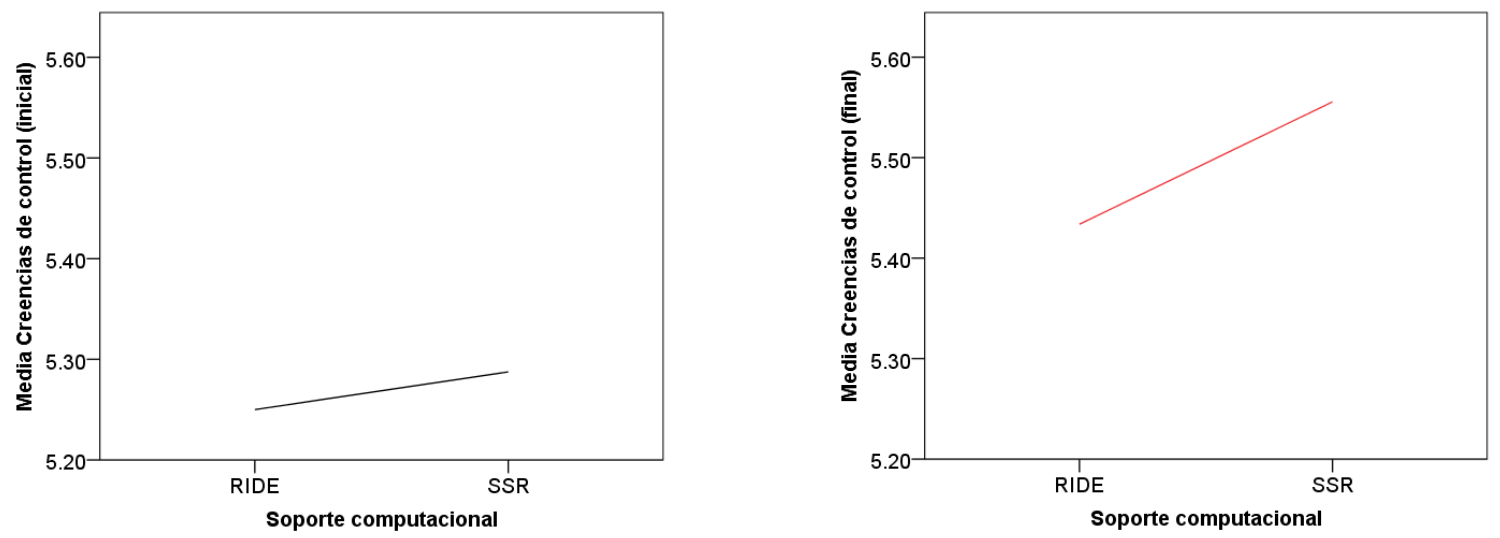

Autorregulación metacognitiva
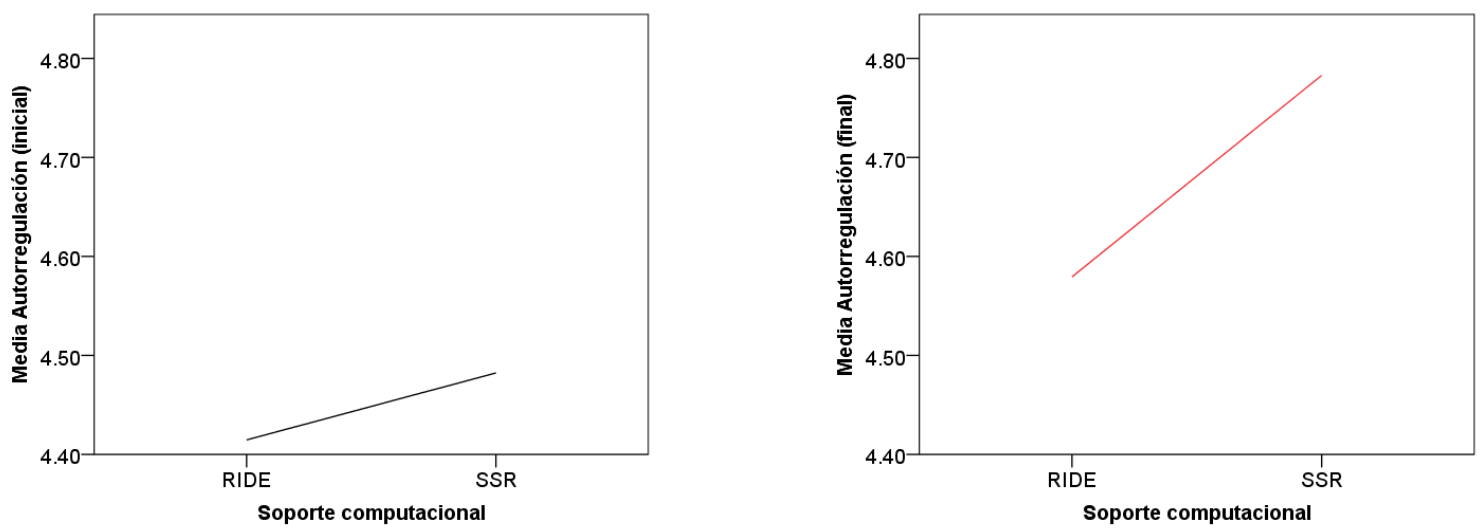

La figura de la izquierda (línea negra) indica el nivel de la variable antes de la intervención.

La figura de la derecha (línea roja) indica el nivel de la variable después de la intervención.

Figura 5. Logro de aprendizaje y autorregulación antes y después de la intervención, dependiendo del soporte computacional para apoyar la regulación social

Fuente: elaboración propia. 
Estos hallazgos son similares a los reportados en otros estudios que han empleado guiones $y$ herramientas de concienciación para apoyar la colaboración, así como los procesos de planeación y reflexión metacognitiva (Lin, 2018; Su et ál., 2018; Wang et ál., 2017; Zheng y Yu, 2015). En efecto, el hecho que los guiones implementados en la condición SSR favorecieran los procesos de planeación y reflexión grupal, decantó en el beneficio del logro de aprendizaje y la autorregulación de los estudiantes (Järvelä et ál., 2016; Zheng et ál., 2017). En la misma línea, las herramientas de concienciación individual y grupal favorecieron el monitoreo metacognitivo, la construcción de conocimientos de alto nivel (Khosa y Volet, 2014), los procesos de planeación compartida y la solución de la tarea (Rogat y LinnenbrinkGarcia, 2011).

En relación con el método de conformación grupal, las medias marginales que arrojó el análisis multivariado de covarianza (figura 6) señalaron que los sujetos que conformaron grupos homogéneos (HM) obtuvieron mejores resultados en el logro de aprendizaje $(\mathrm{M}=63,44, E T=1,60)$, las creencias de control sobre el aprendizaje $(M=5,56, E T=0,08)$ y la autorregulación $(M=4,69, E T=0,07)$. Estos resultados contradicen las investigaciones previas que abogan por la superioridad de los grupos heterogéneos frente a los homogéneos (Johnson y Johnson, 1989; Kagan y Kagan, 2009; Webb et ál., 1997). Una posible explicación al respecto reposa en la propia literatura sobre la regulación interpersonal del aprendizaje, paradigma desde el cual se asume que la metacognición puede ser compartida en la medida que los integrantes del grupo posean estándares similares para el monitoreo metacognitivo, $y$ que cada sujeto tenga cierto grado de similitud en términos de sus habilidades (Malmberg et ál., 2017; Winne, 2015).

En este sentido, el efecto que el ambiente de aprendizaje tuvo sobre los estudiantes, principalmente sobre aquellos asociados en grupos homogéneos, pudo deberse a la implementación de herramientas de concienciación, en función de facilitar la autorregulación y la adaptación metacognitiva (Fransen, Weinberger y Kirschner, 2013), beneficio que en gran parte depende de la existencia de modelos mentales afines, compartidos, que propician la formulación de metas y el desarrollo de habilidades cognitivas y metacognitivas (Zhou y Wang, 2010).

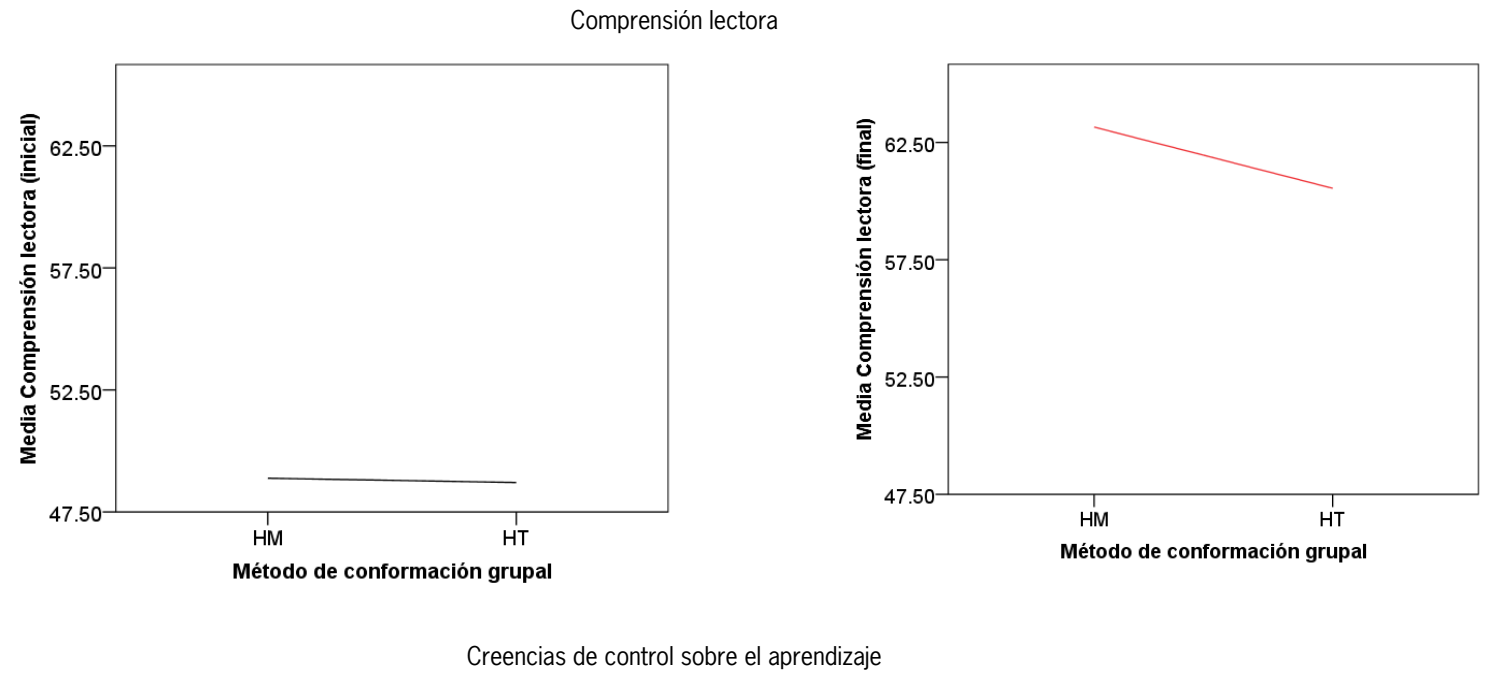





La figura de la izquierda (línea negra) indica el nivel de la variable antes de la intervención.

La figura de la derecha (línea roja) indica el nivel de la variable después de la intervención.

Figura 6. Logro de aprendizaje y autorregulación antes y después de la intervención, dependiendo del método de conformación grupal

Fuente: elaboración propia.

\section{Conclusiones}

El estudio propuesto permitió comparar dos modelos para apoyar la regulación social durante el trabajo colaborativo apoyado por computador, modelos que no habían sido comparados con anterioridad en la literatura sobre regulación interpersonal del aprendizaje. Por supuesto, previamente algunos investigadores ya habían explorado los efectos de la regulación de la tarea y la regulación social, sobre el desempeño de grupos que trabajaban colaborativamente (Janssen, Erkens, Kirschner y Kanselaar, 2012); sin embargo, a la fecha no se habían abordado puntualmente los modelos explorados en nuestro estudio: regulación de la tarea a partir del uso de guiones y herramientas de concienciación (Miller y Hadwin, 2015), en oposición a la regulación de la comunicación y la colaboración con base en el conjunto de reglas RIDE (Saab et ál., 2007).

Nuestros resultados coinciden parcialmente con los hallazgos reportados por Janssen y sus colaboradores (2012), es decir, regular la tarea suele beneficiar en mayor medida el desempeño, cuando se enfoca en fortalecer los procesos de planeación, monitoreo y evaluación durante el trabajo colaborativo. No obstante, trascendiendo estas coincidencias, también encontramos que la regulación de la tarea y la regulación de la colaboración, no solo favorecen el desempeño, sino que además impactan significativamente la autorregulación de los sujetos. Estos resultados sugieren que involucrar ambos tipos de 
regulación en los entornos computacionales, puede favorecer mucho más estas variables dependientes, en la medida que se combinen de manera efectiva, guiones y herramientas de concienciación que promuevan la emergencia y el sostenimiento de comportamientos reguladores durante la colaboración.

Involucrar el método de conformación grupal en nuestro diseño metodológico, también ofrece aportes de valioso interés para las líneas de investigación en regulación social y trabajo colaborativo apoyado por computador. Los hallazgos reportados en este estudio sugieren que la regulación social, sin importar el tipo de actividad que sea objeto de regulación (actividades asociadas con la tarea o con la comunicación y la colaboración), favorece el logro y la autorregulación de sujetos que trabajan tanto en grupos homogéneos como en grupos heterogéneos, teniendo en cuenta conocimientos y habilidades en un dominio específico de conocimiento. Estos resultados atañen no solo al ámbito educativo, sino también al teletrabajo colaborativo acaecido en distintos contextos profesionales, entornos en los que no siempre es posible generar condiciones de heterogeneidad al conformar los equipos de trabajo en los que se involucran sus empleados.

Otra de las fortalezas en nuestro estudio correspondió a la inclusión del estilo cognitivo en la dimensión dependencia/independencia de campo, en el marco de las investigaciones sobre regulación social durante el trabajo colaborativo apoyado por computador. Si bien otros investigadores ya habían considerado estas características individuales en relación con ambientes hipermedia en modalidad b-learning (López et ál., 2012), a la fecha no se había contemplado el estilo cognitivo en relación con modelos de regulación social (regulación de la tarea o regulación de la colaboración), ni métodos de conformación grupal según las habilidades de los estudiantes. En esta dirección, los resultados aquí reportados sugieren que las diferencias que emergen entre sujetos con distinto estilo cognitivo al interactuar en ambientes computacionales pueden reducirse a través de modelos que apoyen la regulación interpersonal del aprendizaje durante la colaboración.

\section{Referencias}

Altun, A. y Cakan, M. (2006). Undergraduate students' academic achievement, field dependent/independent cognitive styles and attitude toward computers. Educational Technology \& Society, 9(1), 289-297.

Angeli, C., Valanides, N. y Kirschner, P. (2009). Field dependence-independence and instructional-design effects on learners' performance with a computer-modeling tool. Computers in Human Behaviour, 25(6), 1355-1366. https://doi. org/10.1016/j.chb.2009.05.010

Armbruster, B., Echols, C. y Brown, A. (1983). The role of metacognition in reading to learn: a developmental perspective. University of Illinois.

Bandura, A. (1986). Social foundations of thought and action. Nueva Jersey: Prentice-Hall.

Bodemer, D. y Dehler, J. (2011). Group awareness in CsCL environments. Computers in Human Behaviour, 27(3), 1043-1045. https://doi.org/10.1016/j. chb.2010.07.014

Bower, G. y Cirilo, R. (1985). Cognitive psychology and text processing. En T. van Dijk (ed.), Handbook of discourse processing (Vol. 1, pp. 71-105). Londres: Academic Press.

Brindley, J., Walti, C. y Blaschke, L. (2009). Creating effective collaborative learning groups in an online environment. International Review of Research in Open and Distance Learning, 10(3), 1-18. https:// doi.org/10.19173/irrodl.v10i3.675

Brown, R., El-Dinary, P., Pressley, M. y Coy-Ogan, L. (1995). A transactional strategies approach to reading instruction. The Reading Teacher, 49(3), 256-258.

Camargo-Uribe, Á. y Hederich-Martínez, C. (2004). Estilo cognitivo y lectura de palabras. Análisis de diferencias en el proceso de acceso al léxico. Folios, 20, 123-137. https://doi.org/10.17227/01234870.20folios123.137

Campbell, D. y Stanley, J. (2005). Diseños experimentales $y$ cuasiexperimentales en la investigación social. Amorrortu Editores.

Cañón, A. y Luna, F. (2011). PISA: comprensión lectora. I. Marco y análisis de los ítems. Instituto Vasco de Evaluación e Investigación Educativa.

Cerchiaro, E., Sánchez, L., Herrera, J., Arbeláez, M. y Gil, H. (2011). Un acercamiento a la metacognición y la comprensión lectora en estudiantes universitarios de México y Colombia. Editorial Unimagdalena. 
Chen, S. y Macredie, R. (2002). Cognitive styles and hypermedia navigation: development of a learning model. Journal of the American Society for Information Science and Technology, 53(1), 3-15. https://doi.org/10.1002/asi.10023

Chiong, R. y Jovanovic, J. (2012). Collaborative learning in online study groups: an evolutionary game theory perspective. Journal of Information Technology Education, 11, 81-101. https://doi.org/10.28945/1574

Cohen, E. (1992). Conditions for productive small groups. Issues in Restructuring Schools, (2), 4-7.

Dillenbourg, P. (1999). What do you mean by 'collaborative learning'? En P. Dillenbourg (ed.), Collaborativelearning: cognitive and computational approaches (pp. 1-19). Oxford: Elsevier.

Dillenbourg, P. (2002). Over-scripting CsCL: The risks of blending collaborative learning with instructional design. En P. Kirschner (ed.), Three worlds of CSCL. Can we support CSCL (pp. 61-91). Heerlen: Open Universiteit Nederland.

Dillenbourg, P., Baker, M., Blaye, A. y O’Malley, C. (1995). The evolution of research on collaborative learning. En E. Spada y P. Reiman, Learning in humans and machine: towards an interdisciplinary learning science (pp. 189-211). Oxford: Elsevier.

Dubouis, M. (2000). El proceso de lectura. Aique.

El-Deghaidy, H. y Nouby, A. (2008). Effectiveness of a blended e-learning cooperative approach in an Egyptian teacher education programme. Computers \& Education, 51(3), 988-1006. https:// doi.org/10.1016/j.compedu.2007.10.001

Falk, D. y Johnson, D. (1977). The effects of perspective-taking and egocentrism on problem solving in heterogeneous and homogeneous groups. The Journal of Social Psychology, 102(1), 63-72. https:// doi.org/10.1080/00224545.1977.9713241

Fiedler, F., Meuwese, W. y Oonk, S. (1961). An exploratory study of group creativity in laboratory tasks. Acta Psychologica, 18, 100-119. https://doi. org/10.1016/0001-6918(61)90008-7

Fransen, J., Weinberger, A. y Kirschner, P. (2013). Team effectiveness and team development in CSCL. Educational Psychologist, 48(1), 9-24. https://doi.org /10.1080/00461520.2012.747947

Gabbs, G. (2009). The assessment of group work: lessons from the literature. Oxford Brookes University. https://www.brookes.ac.uk/aske/documents/
Brookes\%20groupwork\%20Gibbs\%20Dec\%2009. pdf

Garrison, R., Anderson, T. y Archer, W. (2001). Critical thinking and computer conferencing: a model and tool to assess cognitive presence. American Journal of Distance Education, 15(1), 7-23. https://doi. org/10.1080/08923640109527071

Gilly, M. (1989). The psychosocial mechanisms of cognitive constructions: Experimental research and teaching perspectives. International Journal of Educational Research, 13(6), 607-621. https://doi. org/10.1016/0883-0355(89)90044-X

Gokhale, A. (1995). Collaborative learning enhances critical thinking. Journal of Techology Education, 7(1), 22-30. https://doi.org/10.21061/jte.v7i1.a.2

Grabe, W. (2003). Revaloración del término "interactivo". En E. Rodríguez y E. Lager, La lectura (pp. 81-98). Universidad del Valle.

Graham, C. (2005). Blended learning systems. En C. Bonk y C. Graham, The handbook of blended learning: Global perspectives, local designs. Pfeiffer.

Grigorenko, E. y Sternberg, R. (1995). Thinking styles. En D. Saklofske y M. Zeidner, International handbook of personality and intelligence (pp. 205-230). Springer.

Guisande, M., Páramo, M., Tinajero, C. y Almeida, L. (2007). Field dependence-independence (FDI) cognitive style: an analysis of attentional functioning. Psicothema, 19(4), 572-577.

Hadwin, A., Järvelä, S. y Miller, M. (2011). Self-regulated, co-regulated, and socially shared regulation of learning. En B. Zimmerman y D. Schunk (eds.), Handbook of self-regulation of learning and performance (pp. 65-84). Routledge.

Hadwin, A., Järvelä, S. y Miller, H. (2017). Self-regulation, co-regulation and shared regulation in collaborative learning environments. En D. Schunk y G. Greene, Handbook of self-regulation of learning and performance (pp. 83-106). 2a. ed. Londres: Routledge. https://doi.org/10.4324/9781315697048-6

Handal, B. y Herrington, A. (2004). On being dependent or independent in computer based learning environments. UTsepREss. https://files.eric.ed.gov/fulltext/ EJ850352.pdf

Hederich-Martínez, C. (2007). Estilo cognitivo en la dimensión de dependencia-independencia de campo. Influencias culturales e implicaciones educativas. Bogotá: Universidad Pedagógica Nacional. 
Hederich-Martínez, C. (2013). Estilística educativa. Revista Colombiana de Educación, 64, 21-56. https:// doi.org/10.17227/01203916.64rce21.56

Hederich-Martínez, C. y Camargo-Uribe, A. (1999). Estilos cognitivos en Colombia. Universidad Pedagógica Nacional.

Hederich-Martínez, C. y Camargo-Uribe, A. (2000). Estilo cognitivo y logro académico en la ciudad de Bogotá. Revista Colombiana de Educación, (40-41). https://doi.org/10.17227/01203916.7782

Hederich-Martínez, C. y Camargo-Uribe, A. (2016). Cognitive style and educational performance. The case of public schools in Bogotá, Colombia. Psychology, 36(4), 719-737. https://doi.org/10.1080 /01443410.2015.1091916

Hederich-Martínez, C., López-Vargas, O. y CamargoUribe, A. (2016). Effects of the use of a flexible metacognitive scaffolding on self-regulated learning during virtual education. International Journal of Technology Enhanced Learning, 8(3), 199-216. https://doi.org/10.1504/IJTEL.2016.082321

Hilton, S. y Phillips, F. (2010). Instructor-assigned and student-selected groups: a view from inside. Issues in Accounting Education, 25(1), 15-33. https://doi. org/10.2308/iace.2010.25.1.15

Hwang, W., Hsu, J., Shadiev, R. y Chang, C. H. (2015). Employing self-assessment, journaling, and peer sharing to enhance learning from an online course. Journal of Computing in Higher education, 27, 114133. https://doi.org/10.1007/s12528-015-9096-3

Janssen, J. y Bodemer, D. (2013). Coordinated computer-supported collaborative learning: awareness and awareness tools. Educational Psychologist, 48(1), 40-55. https://doi.org/10.1080/00461520.2012.74 9153

Janssen, J., Erkens, G., Kirschner, P. y Kanselaar, G. (2012). Task-related and social regulation during online collaborative learning. Metacognition Learning, 25-43. https://doi.org/10.1007/s11409-010-9061-5

Järvelä, S., Malmberg, J. y Koivuniemi, M. (2016). Recognizing socially shared regulation by using the temporal sequences of online chat and logs in CSCL. Learning and Instruction, 42, 1-11. https://doi. org/10.1016/j.learninstruc.2015.10.006

Järvelä, S., Järvenoja, H., Malmberg, J. y Hadwin, A. (2013). Exploring socially shared regulation in the context of collaboration. Journal of Cognitive
Education and Psychology, 12(3), 267-286. https:// doi.org/10.1891/1945-8959.12.3.267

Jermann, P. (2004). Computer support for interaction regulation in collaborative problem-solving. [Tesis doctoral]. Université de Genève. Ginebra, Suiza.

Johnson, D. y Johnson, R. (1989). Cooperation and competition: theory and research. Edina: Interaction Book Company.

Juwah, C. (2006). Interactions in online peer learning. En R. Sharma y C. Juwah, Interactions in online education. Implications for theory and practice (pp. 171-190). Lawrence Erlbaum.

Kagan, S. y Kagan, M. (2009). Cooperative learning. Kagan Publishing.

Khosa, D. y Volet, S. (2014). Productive group engagement in cognitive activity and metacognitive regulation during collaborative learning: Can it explain differences in students' conceptual understanding? Metacognition and Learning, 9, 287-307. https://doi. org/10.1007/s11409-014-9117-Z

Kintsch, W. y Van Dijk, T. A. (1983). Strategies of discourse comprehension. Nueva York: Academic Press.

Kirchner, T. (1987). Estilo cognitivo de "dependencia independencia de campo" y proceso lector. [Tesis de doctorado]. Universitat de Barcelona. Barcelona, España.

Kirschner, P. y Erkens, G. (2013). Toward a Framework for CSCL Research. Educational Psychologist, 48(1), 1-8. https://doi.org/10.1080/00461520.2012.750227

Kirschner, P., Kreijns, K. y Fransen, J. (2014). Awareness of cognitive and social behavior in a CSCL environment. Journal of Computer Assisted Learning, 31(1), 59-77. https://doi.org/10.1111/jcal.12084

Kirschner, F., Paas, F. y Kirschner, P. (2011). Task complexity as a driver for collaborative learning efficiency: the collective working-memory effect. Applied Cognitive Psychology, 25(4), 615-624. https:// doi.org/10.1002/acp.1730

Kirschner, P., Sweller, J., Kirschner, F. y Zambrano, J. (2018). From cognitive load theory to collaborative cognitive load theory. International Journal of Computer-Supported Collaborative Learning, 13, 213-233. https://doi.org/10.1007/s11412-018-9277-y

Kozhevnikov, M. (2007). Cognitive styles in the context of modern psychology: toward an integrated framework of cognitive style. Psychological Bulletin, 133(3), 464481. https://doi.org/10.1037/0033-2909.133.3.464 
Kreijns, K., Kirschner, P. y Jochems, W. (2003). Identifying the pitfalls for social interaction in computer-supported collaborative learning environments: a review of the research. Computers in Human Behavior, 19(3), 335-353. https://doi. org/10.1016/S0747-5632(02)00057-2

Lai, E. (2011). Collaboration: a literature review. Pearson. http://images.pearsonassessments.com/images/ tmrs/Collaboration-Review.pdf

Lin, J. (2018). Effects of an online team project-based learning environment with group awareness and peer evaluation on socially shared regulation of learning and self-regulated learning. Behaviour \& Information Technology, 37(5), 445-461. https://doi. org/10.1080/0144929X.2018.1451558

López, O. (2010). Aprendizaje autorregulado, estilo cognitivo y logro académico en ambientes computacionales. [Tesis doctoral]. Universidad Pedagógica Nacional. Bogotá, Colombia

López, O., Hederich-Martínez, C. y Camargo-Uribe, Á. (2012). Logro de aprendizaje en ambientes hipermediales: andamiaje autorregulador y estilo cognitivo. Revista Colombiana de Educación, 44(2), 13-26.

Malmberg, J., Järvelä, S. y Järvenoja, H. (2017). Capturing temporal and sequential patterns of self-, co-, and socially shared regulation in the context of collaborative learning. Contemporary Educational Psychology, 49, 160-174. https://doi.org/10.1016/j. cedpsych.2017.01.009

Manlove, S., Lazonder, A. y Jong, T. d. (2006). Regulative support for collaborative scientific inquiry learning. Journal of Computer Assisted Learning, 22(2), 87-98. https://doi.org/10.1111/j.1365-2729.2006.00162.x

Martens, K. (1975). Cognitive style: an introduction with annotated bibliography. En American College Personnel Association Convention (pp. 1-18). State University of New York.

Masterman, L. y Sharples, M. (2002). A theory-informed framework for designing software to support reasoning about causation in history. Computers \& Education, 38, 165-185. https://doi.org/10.1016/ S0360-1315(01)00072-0

McCaslin, M. y Good, T. (1996). Listening in classrooms. Harper Collins Publishers Inc.

Mertler, C. y Vannatta, R. (2017). Advanced and multivariate statistical methods. Routledge.
Messick, S. (1967). The criterion problem in the evaluation of instruction: assessing possible not just intended outcomes. University of California.

Miller, A. (1987). Cognitive styles: an integrated model. Educational Psychology, 7(4), 251-268. https://doi. org/10.1080/0144341870070401

Miller, M. y Hadwin, A. (2015). Scripting and awareness tools for regulating collaborative learning: changing the landscape of support in CSCL. Computers in Human Behavior, 52, 573-588. https://doi. org/10.1016/j.chb.2015.01.050

Ministerio de Educación Nacional (MEN). (2019). Supérate. Bogotá. Recuperado de https://www.mineducacion.gov.co/portal/Preescolar-basica-y-media/ Proyectos-de-Calidad/339326:Superate

Myint, S. (1996). The interaction of cognitive styles with varying levels of feedback in multimedia presentation. International Journal of instructional Media, 23(3), 229-237.

O’Donell, A. y Dansereau, D. (1992). Scripted cooperation in student dyads: a method for analyzing and enhancing academic learning and performance. En R. Hertz-Lazarowitz y N. Miller, Interaction in cooperative groups. The theoretical anatomy of group learning (pp. 120-141). Cambridge University Press.

Organización para la Cooperación y el Desarrollo Económicos (OECD). (2010). PISA 2009 Results: what students know and can do - Student performance in reading, mathematics and science. París. https://doi. org/10.1787/9789264091450-en

Organización para la Cooperación y el Desarrollo Económicos (OECD) (2015). PISA 2015 Results. Excellence and equity in education. Vol. 1. París.

Paivio, A. (1990). Mental representations. A dual coding approach. Oxford University Press. https://doi. org/10.1093/acprof:oso/9780195066661.001.0001

Panadero, E., Kirschner, P., Järvelä, S., Malmberg, J. y Järvenoja, H. (2015). How individual self-regulation affects group regulation and performance: A shared regulation intervention. Small Group Research, 46(4), 431-454. https://doi.org/10.1177/1046496415591219

Pea, R. (1994). Learning scientific concepts through material and social activities: conversational analysis meets conceptual change. Educational Psychologist, 28(3), 265-277. https://doi.org/ $10.1207 /$ s15326985ep2803_6 
Perera, D., Kay, J., Koprinska, I., Yacef, K. y Zaïane, O. (2009). Clustering and sequential pattern mining of online collaborative learning data. IEEE Transactions on Knowledge and Data Engineering, 21(6), 759-772. https://doi.org/ 10.1109/TKDE.2008.138

Pijls, M., Dekker, R. y Van Hout-Wolters, B. (2003). Mathematical level raising through collaborative investigations with the computer. International Journal of Computers for Mathematical Learning, 8, 191-213. https://doi. org/10.1023/B:IJCO.0000003874.51843.87

Pintrich, P., Smith, D., García, T. y McKeachie, W. (1991). A manual for the use of the motivated strategies for learning questionnaire (MSLQ). The University of Michigan.

Pi-Sui-Hsu y Dwyer, F. (2004). Effect of level of adjunct questions on achievement of field independent/ field dependent learners. International Journal of Instructional Media, 31(1), 99-105.

Pressley, M. (2002). Metacognition and self-regulated comprehension. En A. Farstrup y J. Samuels, What research has to say about reading instruction (pp. 291-309). 3a. ed. International Reading Association. https://doi.org/10.1598/0872071774.13

Rogat, T. y Linnenbrink-García, L. (2011). Socially shared regulation in collaborative groups: an analysis of the interplay between quality of social regulation and group processes. Cognition and Instruction, 29(4), 375-415. https://doi.org/10.1080/07370008. 2011.607930

Roschelle, J. (1992). Learning by collaborating: convergent conceptual change. Journal of the Learning Sciences, 2(3), 235-276. https://doi.org/10.1207/ s15327809jls0203_1

Roschelle, J. y Teasley, S. (1995). The construction of shared knowledge in collaborative problem solving. En C. O'Malley, Computer Supported Collaborative Learning (pp. 69-97). Springer-Verlag.

Rumelhart, D. (2003). Hacia una comprensión de la comprensión. En E. Rodríguez y E. Lager, La lectura (pp. 25-52). Universidad del Valle.

Rumelhart, D. y Norman, D. (1978). Accretion, tuning, and reestructuring: three modes of learning. En J. Cotton y L. Klatsky, Semantic factors in cognition (pp. 37-53). Lawrence Erlbaum, Hillsdale.

Saab, N., Van Joolingen, W. y Van Hout-Wolters, B. (2005). Communication in collaborative discovery learning.
British Journal of Educational Psychology, 15(4), 603-621. https://doi.org/10.1348/000709905X42905

Saab, N., Van Joolingen, W. y Van Hout-Wolters, B. (2007). Supporting communication in a collaborative discovery learning environment: the effect of instruction. Instructional Science, 35, 73-98. https:// doi.org/10.1007/s11251-006-9003-4

Sabet, M. y Mohammadi, S. (2013). The relationship between field independence/dependence styles and reading comprehension abilities of EFL readers. Theory and Practice in Language Studies, 3(11), 21412150. https://doi.org/10.4304/tpls.3.11.2141-2150

Salmani-Nodoushan, M. (2007). Is field dependence or independence a predictor of EFL reading performance? TESL Canada Journal, 24(2), 82-108. https:// doi.org/10.18806/tesl.v24i2.140

Santiago, Á., Castillo, M. y Ruiz, J. (2010). Lectura, metacognición y evaluación. Alejandría.

Sawa, H. (1966). Analytic thinking and synthetic thinking. Bulletin of Faculty of Education, 13, 1-16.

Schoor, C. y Bannert, M. (2012). Exploring regulatory processes during a computer-supported collaborative learning task using process mining. Computers in Human Behavior, 28(4), 1321-1331. https://doi. org/10.1016/j.chb.2012.02.016

Secretaría de Educación del Distrito (SED) (2015). Estrategia A-probar. Bogotá. https://www.educacionbogota.edu.co/portal_institucional/node/5853

Shete, S., Beasley, T., Etzel, C., Fernández, J., Chen, J., Allison, D. y Amos, C. (2004). Effect of winzorizarion on power and type 1 error of variance components and related methods of qtl detection. Behavior Genetics, 34, 153-159. https://doi.org/10.1023/B:BEGE.0000013729.26354.da

Su, Y., Li, Y., Hu, H. y Rosé, C. (2018). Exploring college english language learners' self and social regulation of learning during wiki-supported collaborative reading activities. International Journal of ComputerSupported Collaborative Learning, 13, 35-60. https:// doi.org/10.1007/s11412-018-9269-y

Suh, J. S. (2009). Role of field independence-dependence in EFL reading. Contemporary Grammar Research, 58, 203-220. http://dspace.kci.go.kr/handle/ $\mathrm{kci} / 283153$

Tabanachi, B. y Fidell, L. (2014). Using multivariate statistics. Pearson. 
Tardif, J. (2003). La evaluación del saber-leer: un asunto más de competencia que de actuación. En E. Rodríguez y E. Lager, La lectura (pp. 165-194). Universidad del Valle.

The Metiri Group (2009). The impact of collaborative, scaffolded learning in K-12 schools: a meta-analysis. Cisco. http://docshare01.docshare. tips/files/7943/79437698.pdf

Tinajero, C., Lemos, S., Araújo, M., Ferraces, M. y Páramo, M. (2012). Cognitive style and learning strategies as factors which affect academic achievement of brazilian university students. Psicologia, 25(1), 105-113. https://doi.org/10.1590/ S0102-79722012000100013

Trilling, B. y Fadel, C. (2009). 21st century skills: learning for life in our times. Jossey-Bass.

Van Dijk, T. (1992). La ciencia del texto. Paidós.

Vauras, M. y Volet, S. (2013). The study of interpersonal regulation in learning and its challenge to the research methodology. En S. Volet y M. Vauras (eds.), Interpersonal regulation of learning and motivation (pp. 1-13). Routledge.

Volet, S., Summers, M. y Thurman, J. (2009). Highlevel co-regulation in collaborative learning: How does it emerge and how is it sustained? Learning and Instruction, 19(2), 128-143. https://doi. org/10.1016/j.learninstruc.2008.03.001

Volkmar, F. (2013). Encyclopedia of autism spectrum disorders. Springer.

Wang, X., Kollar, I. y Stegmann, K. (2017). Adaptable scripting to foster regulation processes and skills in computer-supported collaborative learning. International Journal of Computer-Supported Collaborative Learning, 12, 153-172. https://doi. org/10.1007/s11412-017-9254-x

Wasson, B. (1998). Identifying coordination agents for collaborative telelearning. International Journal of Artificial Intelligence in Education, 9, 275-299.

Webb, N., Nemer, K. y Chizhik, A. (1997). Equity issues in collaborative group assessment: group composition and performance. University of California.

Weller, H., Repman, J. y Rooze, G. (1994). The relationship of learning, behavior, and cognitive style in hypermedia-based instruction. Computers in the schools. Interdisciplinary Journal of Practice, Theory, and Applied Research, 10(3-4), 401-418. https://doi. org/10.1300/J025v10n03_09

Winne, P. (2015). What is the state of the art in self-, co-and socially shared regulation in CSCL? Computers in Human Behavior, 52, 628-631. https:// doi.org/10.1016/j.chb.2015.05.007

Winne, P. y Hadwin, A. (1998). Studying as self-regulated learning. Metacognition in Educational Theory and Practice, 93, 27-30.

Winne, P. y Hadwin, A. (2008). The weave of motivation and self-regulated learning. En D. Schunk y B. Zimmerman, Motivation and self-regulated learning: theory, research, and applications (pp. 297-314). Lawrence Erlbaum Associates.

Witkin, H. y Asch, S. (1948). Studies in space orientation. III. Perception of the upright in the absence of a visual field. Journal of Experimental Psychology, 38(5), 603-614. https://doi.org/10.1037/h0055372

Witkin, H. y Goodenough, D. (1977). Field dependence and interpersonal behavior. Psychologica Bulletin, 84(4), 661-689. https://doi. org/10.1037/0033-2909.84.4.661

Zehavi, N. (1995). Integrating software development with research and teacher education. Computers in the Schools, 11(3), 11-24. https://doi.org/10.1300/ J025v11n03_03

Zhang, L., Kalyuga, S., Lee, C. y Lei, C. (2016). Effectiveness of collaborative learning of computer programming under different learning group formations according to students' prior knowledge: a cognitive load perspective. Journal of Interactive Learning Research, 29(2), 171-192.

Zheng, L. y Huang, R. (2016). The effects of sentiments and co-regulation on group performance in computer supported collaborative learning. The Internet and Higher Education, 28, 59-67. https:// doi.org/10.1016/j.iheduc.2015.10.001

Zheng, L. y Yu, J. (2015). The empirical study on self-regulation, co-regulation, and socially shared regulation in computer-supported collaborative learning. En 2015 IEEE 15th International Conference on Advanced Learning Technologies (ICALT) (pp. 180-184). https://doi.org/10.1109/ICALT.2015.37

Zheng, L., Li, X. y Huang, R. (2017). The effect of socially shared regulation approach on learning performance 
in computer-supported collaborative learning. Journal of Educational Technology \& Society, 20(4), 35-46.

Zhou, Y. y Wang, E. (2010). Shared mental models as moderators of team process-performance relationships. Social Behavior and Personality, 38(4), 433-444. https://doi.org/10.2224/sbp.2010.38.4.433
Ziller, R. (1955). Scales of judgement: a determinant of the accuracy of group decisions. Human Relations, 8(2), 153-164. https://doi. org/10.1177/001872675500800203

Zimmerman, B. (1989). Social cognitive view of self-regulated academic learning. Journal of Educational Psychology, 81(3), 329-339. https://doi. org/10.1037/0022-0663.81.3.329 